\title{
THE LOW-DIMENSIONAL ALGEBRAIC COHOMOLOGY OF THE WITT AND THE VIRASORO ALGEBRA WITH VALUES IN NATURAL MODULES
}

\author{
JILL ECKER AND MARTIN SCHLICHENMAIER
}

\begin{abstract}
The main aim of this contribution is to compute the low-dimensional algebraic cohomology of the Witt and the Virasoro algebra with values in the adjoint and the trivial module. The last section includes results for the general tensor densities modules, presented without proof. One of our main results is that the third algebraic cohomology of the Witt algebra with values in the adjoint module vanishes, while it is one-dimensional for the Virasoro algebra. The first and the second algebraic cohomology of the Witt and the Virasoro algebra with values in tensor densities modules vanish for almost all modules. In the case they do not vanish, we give explicit expressions for the generating cocycles. In our work, we consider algebraic cohomology and not only the sub-complex of continuous cohomology, meaning we do not put any continuity constraints on the cochains. Consequently, our results are independent of any choice of an underlying topology, and valid for any concrete realizations of the considered Lie algebras.
\end{abstract}

\section{INTRODUCTION}

The Witt algebra and its universal central extension, the Virasoro algebra, are two of the most important infinite-dimensional Lie algebras, as they have many applications both in mathematics and theoretical physics. The Virasoro algebra is omnipresent in string theory, where it is attached to physical observables such as the mass spectrum. In two-dimensional conformal field theory, the Virasoro algebra is of outermost importance.

The cohomology of Lie algebras and the low-dimensional cohomology in particular, has numerous interpretations in terms of known objects such as invariants, outer derivations, extensions, deformations and obstructions, as well as crossed modules, see e.g. Gerstenhaber [13 17. The analysis of these objects leads to a better understanding of the Lie algebra itself. Moreover, deformations of Lie algebras can yield families of new Lie algebras.

In the so-called continuous cohomology, many results about the classical infinite-dimensional Lie algebras such as the Witt algebra are known. In fact, a geometrical realization of the Witt algebra is given by the complexified Lie algebra of polynomial vector fields on the circle, which forms a dense subalgebra of the complexified Lie algebra of smooth vector fields on the

Date: 13.12 .2019 .

2000 Mathematics Subject Classification. Primary: 17B56; Secondary: 17B68, 17B65, 17B66, 14D15, 81R10, 81T40.

Key words and phrases. Witt algebra; Virasoro algebra; Lie algebra cohomology; Deformations of algebras; conformal field theory.

Partial support by the Internal Research Project GEOMQ15, University of Luxembourg, and by the OPEN programme of the Fonds National de la Recherche (FNR), Luxembourg, project QUANTMOD O13/570706 is gratefully acknowledged. 
circle, $\operatorname{Vect}\left(S^{1}\right)$. In this setting, it is natural to consider continuous cohomology. The continuous cohomology of vector fields on the circle with values in the trivial module is known, see the results by Gelfand and Fuks [11, 12. Similarly, based on results of Goncharova [18, Reshetnikov [24 and Tsujishita [27, the vanishing of the continuous cohomology of vector fields on the circle with values in general tensor densities modules, which include the adjoint module, was proved by Fialowski and Schlichenmaier in [10.

Less is known about the so-called algebraic cohomology, also known as discrete cohomology, which contains the continuous cohomology as a sub-complex. The primary definition of the Witt and the Virasoro algebra is based on the Lie structure and is thus purely algebraic. In this article, we consider the Witt and the Virasoro algebra as purely algebraic objects, and we do not work in specific geometrical realizations of these. Hence, our results are independent of any underlying topology chosen. Similarly, our cochains are purely algebraic cochains, meaning we do not restrict ourselves to continuous cochains. Actually, there are limitations for the continuous cohomology of purely algebraic infinite-dimensional Lie algebras, see e.g. Wagemann [30]. Moreover, algebraic cohomology works for any base field $\mathbb{K}$ with characteristic zero, and not only for the fields $\mathbb{C}$ or $\mathbb{R}$. Therefore, knowledge of the algebraic cohomology is needed.

In the literature, results on the algebraic cohomology of the Witt and the Virasoro algebra are somewhat scarce. In fact, algebraic cohomology is in general much harder to compute than continuous cohomology. The vanishing of the second algebraic cohomology of the Witt and the Virasoro algebra with values in the adjoint module was shown almost at the same time independently by Schlichenmaier [25, 26] and Fialowski [9], by using elementary algebraic methods. Without proof, Fialowski announced the result for the Witt algebra already in [8]. Van den Hijligenberg and Kotchetkov proved in [28] the vanishing of the second algebraic cohomology with values in the adjoint module of the superalgebras $k(1), k^{+}(1)$ and of their central extensions.

The main goal of this contribution is to compute the third algebraic cohomology of the Witt and the Virasoro algebra with values in the adjoint module, the case of the trivial module being obtained as a by-product. For reasons of completeness, and also as a warm-up example, we included the computation of the first algebraic cohomology in the appendix. We will also provide results for the general tensor densities modules. Some of the results proven here have been presented as preprints in 2017 and 2018 [5, 6]. This document unites them and should replace them. The results have also been announced in the proceeding [7]. Furthermore, results on general tensor densities modules $\mathcal{F}^{\lambda}$ are added, though without proofs, as the used techniques are quite similar to the ones presented in this document, see [4]. In the proofs, we use both elementary algebraic manipulations and higher tools from algebraic cohomology, such as long exact sequences and spectral sequences.

This article is organized as follows: In Section 2, we recall the algebraic definitions of the Witt and the Virasoro algebra.

In Section 3, we introduce the cohomology of Lie algebras, as well as some tools used to compute this cohomology. This section also contains a brief summary of the results known of the algebraic cohomology of the Witt and the Virasoro algebra for the adjoint and the trivial modules, including the results derived in this contribution.

The Sections 4 and 5 constitute the main part of the present article and contain the proofs related to the third algebraic cohomology of the Witt and the Virasoro algebra with values 
in the adjoint and trivial modules. More precisely, in Section 4 we prove $\mathrm{H}^{3}(\mathcal{W}, \mathcal{W})=\{0\}$. In Section 5 , we start by showing that $\mathrm{H}^{3}(\mathcal{V}, \mathcal{V}) \cong \mathrm{H}^{3}(\mathcal{V}, \mathbb{K})$ by using long exact sequences and spectral sequences. Subsequently, we show that $\mathrm{H}^{3}(\mathcal{V}, \mathbb{K})$ and simultaneously $\mathrm{H}^{3}(\mathcal{W}, \mathbb{K})$ are one-dimensional. We identify a non-trivial 3-cocycle which we call algebraic GodbillonVey cocycle. By essentially elementary but nevertheless intricate algebraic methods one can show that this cocycle is a generator of $\mathrm{H}^{3}(\mathcal{V}, \mathbb{K})$ and $\mathrm{H}^{3}(\mathcal{W}, \mathbb{K})$. Recurrence relations are needed to obtain this result, not unlike those used to prove $\mathrm{H}^{3}(\mathcal{W}, \mathcal{W})=\{0\}$. Thus, we only give parts of the proof. The details can be found ${ }^{1}$ in [4. All in all, we obtain that $\operatorname{dim}\left(\mathrm{H}^{3}(\mathcal{V}, \mathcal{V})\right)=1$ whereas $\mathrm{H}^{3}(\mathcal{W}, \mathcal{W})=\{0\}$. The reader should compare this with the results obtained for the second cohomology, where we have $\mathrm{H}^{2}(\mathcal{W}, \mathcal{W})=\mathrm{H}^{2}(\mathcal{V}, \mathcal{V})=\{0\}$, see [26], [9].

In Section 6, we give the results for the first and second algebraic cohomology of the Witt and the Virasoro algebra with values in the general tensor densities modules, though without proofs. The proofs will be given in [4].

Finally, the appendix contains the computations for the first algebraic cohomology of the Witt and the Virasoro algebra. They are included for completeness, as the results are needed in some proofs. Moreover, they provide an introduction to our computation methods.

\section{The Witt and the Virasoro Algebra}

The Witt algebra $\mathcal{W}$ is an infinite-dimensional, $\mathbb{Z}$-graded Lie algebra first introduced by Cartan in 1909 [2]. As a vector space, the Witt algebra is generated over a base field $\mathbb{K}$ with characteristic zero by the basis elements $\left\{e_{n} \mid n \in \mathbb{Z}\right\}$, which satisfy the following Lie algebra structure equation:

$$
\left[e_{n}, e_{m}\right]=(m-n) e_{n+m}, \quad n, m \in \mathbb{Z} .
$$

The Witt algebra is a $\mathbb{Z}$-graded Lie algebra, the degree of an element $e_{n}$ being defined by $\operatorname{deg}\left(e_{n}\right):=n$. More precisely, the Witt algebra is an internally $\mathbb{Z}$-graded Lie algebra, as the grading is given by one of its own elements, namely $e_{0}:\left[e_{0}, e_{m}\right]=m e_{m}=\operatorname{deg}\left(e_{m}\right) e_{m}$. The Witt algebra $\mathcal{W}$ can thus be decomposed into an infinite sum of one-dimensional homogeneous subspaces $\mathcal{W}_{n}$, where each subspace $\mathcal{W}_{n}$ is generated over $\mathbb{K}$ by a single element $e_{n}$.

The Witt algebra comes with three popular concrete realizations. Algebraically, the Witt algebra can be realized as the Lie algebra of derivations of the infinite-dimensional associative $\mathbb{K}$-algebra of Laurent polynomials $\mathbb{K}\left[Z^{-1}, Z\right]$. A geometrical realization of the Witt algebra is obtained by considering $\mathbb{K}=\mathbb{C}$, which corresponds to the algebra of meromorphic vector fields on the Riemann sphere $\mathbb{C P}^{1}$ that are holomorphic outside of 0 and $\infty$. In this realization, the basis elements of the Witt algebra can be written as $e_{n}=z^{n+1} \frac{d}{d z}$, where $z$ corresponds to the quasi-global complex coordinate. Finally, another geometrical realization of the Witt algebra is given by the complexified Lie algebra of polynomial vector fields on the circle $S^{1}$, in which case the generators are given by $e_{n}=e^{i n \varphi} \frac{d}{d \varphi}$, where $\varphi$ is the coordinate along $S^{1}$.

\footnotetext{
1 or in 6
} 
It is a well-known fact that the Witt algebra, up to equivalence and rescaling, has a unique non-trivial central extension $\mathcal{V}$, which in fact is a universal central extension:

$$
0 \longrightarrow \mathbb{K} \stackrel{i}{\longrightarrow} \mathcal{V} \stackrel{\pi}{\longrightarrow} \mathcal{W} \longrightarrow 0,
$$

where $\mathbb{K}$ is in the center of $\mathcal{V}$. This extension $\mathcal{V}$ is called the Virasoro algebra. Via this extension, all modules of the Witt algebra become in an canonical way also modules of the Virasoro algebra as well as $\mathbb{K}$-modules.

As a vector space, $\mathcal{V}$ is given as a direct sum $\mathcal{V}=\mathbb{K} \oplus \mathcal{W}$, with generators $\hat{e}_{n}:=\left(0, e_{n}\right)$ and the one-dimensional central element $t:=(1,0)$. The generators fulfill the following Lie structure equation:

$$
\begin{aligned}
& {\left[\hat{e}_{n}, \hat{e}_{m}\right]=(m-n) \hat{e}_{n+m}+\alpha\left(e_{n}, e_{m}\right) \cdot t \quad n, m \in \mathbb{Z},} \\
& {\left[\hat{e}_{n}, t\right]=[t, t]=0,}
\end{aligned}
$$

where $\alpha \in Z^{2}(\mathcal{W}, \mathbb{K})$ is the so-called Virasoro 2-cocycle, sometimes also called the GelfandFuks cocycle, which can be represented by:

$$
\alpha\left(e_{n}, e_{m}\right)=-\frac{1}{12}\left(n^{3}-n\right) \delta_{n+m, 0} .
$$

The cubic term $n^{3}$ is the most important term, while the linear term $n$ is a coboundary $2^{2}$ By defining $\operatorname{deg}\left(\hat{e}_{n}\right):=\operatorname{deg}\left(e_{n}\right)=n$ and $\operatorname{deg}(t):=0$, the Virasoro algebra becomes also an internally $\mathbb{Z}$-graded Lie algebra.

\section{The cohomology of Lie algebras}

3.1. The Chevalley-Eilenberg cohomology. For the convenience of the reader, we will briefly recall the Chevalley-Eilenberg cohomology, i.e. the cohomology of Lie algebras.

Let $\mathcal{L}$ be a Lie algebra and $M$ an $\mathcal{L}$-module. We denote by $C^{q}(\mathcal{L}, M)$ the space of $q$ multilinear alternating maps on $\mathcal{L}$ with values in $M$,

$$
C^{q}(\mathcal{L}, M):=\operatorname{Hom}_{\mathbb{K}}\left(\wedge^{q} \mathcal{L}, M\right) .
$$

Elements of $C^{q}(\mathcal{L}, M)$ are called $q$-cochains. By convention, we have $C^{0}(\mathcal{L}, M):=M$. The coboundary operators $\delta_{q}$ are defined by:

$$
\begin{aligned}
\forall q \in \mathbb{N}, \quad & \delta_{q}: C^{q}(\mathcal{L}, M) \rightarrow C^{q+1}(\mathcal{L}, M): \psi \mapsto \delta_{q} \psi, \\
\left(\delta_{q} \psi\right)\left(x_{1}, \ldots x_{q+1}\right): \quad & =\sum_{1 \leq i<j \leq q+1}(-1)^{i+j+1} \psi\left(\left[x_{i}, x_{j}\right], x_{1}, \ldots, \hat{x}_{i}, \ldots, \hat{x}_{j}, \ldots, x_{q+1}\right) \\
& +\sum_{i=1}^{q+1}(-1)^{i} x_{i} \cdot \psi\left(x_{1}, \ldots, \hat{x}_{i}, \ldots, x_{q+1}\right),
\end{aligned}
$$

with $x_{1}, \ldots, x_{q+1} \in \mathcal{L}, \hat{x}_{i}$ means that the entry $x_{i}$ is omitted and the dot $\cdot$ stands for the module structure. For $x \in \mathcal{L}$ and $y \in M$ we have $x \cdot y=[x, y]$ in case of the adjoint module $M=\mathcal{L}$, and $x \cdot y=0$ in case of the trivial module $M=\mathbb{K}$. The coboundary operators satisfy $\delta_{q+1} \circ \delta_{q}=0 \forall q \in \mathbb{N}$, meaning we obtain a cochain complex $\left(C^{*}(\mathcal{L}, M), \delta\right)$ called the Chevalley-Eilenberg complex. The corresponding cohomology is the Chevalley-Eilenberg cohomology defined by:

$$
\mathrm{H}^{q}(\mathcal{L}, M):=Z^{q}(\mathcal{L}, M) / B^{q}(\mathcal{L}, M),
$$

\footnotetext{
2 The symbol $\delta_{i, j}$ is the Kronecker Delta, defined as being one if $i=j$ and zero otherwise.
} 
where $Z^{q}(\mathcal{L}, M):=\operatorname{ker} \delta_{q}$ is the vector space of $q$-cocycles and $B^{q}(\mathcal{L}, M):=\operatorname{im} \delta_{q-1}$ is the vector space of $q$-coboundaries. For more details, we refer the reader to the original literature by Chevalley and Eilenberg [3].

3.2. Degree of a homogeneous cochain. Let $\mathcal{L}$ be a $\mathbb{Z}$-graded Lie algebra $\mathcal{L}=\bigoplus_{n \in \mathbb{Z}} \mathcal{L}_{n}$ and $M$ a $\mathbb{Z}$-graded $\mathcal{L}$-module, i.e. $M=\bigoplus_{n \in \mathbb{Z}} M_{n}$. A $q$-cochain $\psi$ is homogeneous of degree $d$ if there exists a $d \in \mathbb{Z}$ such that for all $q$-tuple $x_{1}, \ldots, x_{q}$ of homogeneous elements $x_{i} \in$ $\mathcal{L}_{\operatorname{deg}\left(x_{i}\right)}$, we have:

$$
\psi\left(x_{1}, \ldots, x_{q}\right) \in M_{n} \text { with } n=\sum_{i=1}^{q} \operatorname{deg}\left(x_{i}\right)+d .
$$

This leads to the decomposition of the cohomology for all $q$ :

$$
\mathrm{H}^{\mathrm{q}}(\mathcal{L}, M)=\bigoplus_{d \in \mathbb{Z}} \mathrm{H}_{(d)}^{q}(\mathcal{L}, M) .
$$

An important result by Fuks [11] states that for internally graded Lie algebras and modules, the cohomology reduces to the degree-zero cohomology:

$$
\begin{aligned}
& \mathrm{H}_{(d)}^{q}(\mathcal{L}, M)=\{0\} \quad \text { for } d \neq 0, \\
& \mathrm{H}^{q}(\mathcal{L}, M)=\mathrm{H}_{(0)}^{q}(\mathcal{L}, M) .
\end{aligned}
$$

3.3. Results on the algebraic cohomology of the Witt and the Virasoro algebra. For future reference, we briefly summarize in this section known results on the algebraic cohomology of the Witt and the Virasoro algebra with values in the adjoint and trivial modules, including the results derived in the present article.

For the zeroth cohomology corresponding to invariants, we immediately obtain by direct computation the following results for the Witt and the Virasoro algebra:

$$
\begin{array}{rll}
\mathrm{H}^{0}(\mathcal{W}, \mathbb{K})=\mathbb{K} & \text { and } & \mathrm{H}^{0}(\mathcal{W}, \mathcal{W})=\{0\}, \\
\mathrm{H}^{0}(\mathcal{V}, \mathbb{K})=\mathbb{K} & \text { and } & \mathrm{H}^{0}(\mathcal{V}, \mathcal{V})=\mathbb{K} t
\end{array}
$$

where $t$ is the central element.

As shown in the appendix and in [4], the first algebraic cohomology of the Witt and the Virasoro algebra is given by:

$$
\begin{aligned}
\mathrm{H}^{1}(\mathcal{W}, \mathbb{K}) & =\{0\} & \text { and } & & \mathrm{H}^{1}(\mathcal{W}, \mathcal{W})=\{0\}, \\
\mathrm{H}^{1}(\mathcal{V}, \mathbb{K}) & =\{0\} & \text { and } & & \mathrm{H}^{1}(\mathcal{V}, \mathcal{V})=\{0\}
\end{aligned}
$$

Concerning the second cohomology related to central extensions and deformations, we have the following results:

$$
\begin{array}{rll}
\operatorname{dim}\left(\mathrm{H}^{2}(\mathcal{W}, \mathbb{K})\right)=1 & \text { and } & \mathrm{H}^{2}(\mathcal{W}, \mathcal{W})=\{0\} \\
\mathrm{H}^{2}(\mathcal{V}, \mathbb{K})=\{0\} & \text { and } & \mathrm{H}^{2}(\mathcal{V}, \mathcal{V})=\{0\}
\end{array}
$$

The first result $\operatorname{dim}\left(\mathrm{H}^{2}(\mathcal{W}, \mathbb{K})\right)=1$ is a well-known result. It states that the Witt algebra admits, up to equivalence and rescaling, only one non-trivial central extension, namely the Virasoro algebra. For an algebraic proof of this result, see e.g. [1, 21]. The second result $\mathrm{H}^{2}(\mathcal{W}, \mathcal{W})=\{0\}$ was announced by Fialowski $[8$, without proof and was shown algebraically by Schlichenmaier [25, 26] and Fialowski [9. This result implies that the Witt algebra is infinitesimally and formally rigid. The third result $\mathrm{H}^{2}(\mathcal{V}, \mathbb{K})=\{0\}$ and the fourth result 
$\mathrm{H}^{2}(\mathcal{V}, \mathcal{V})=\{0\}$ were shown by Schlichenmaier [26].

Concerning the third cohomology related to crossed modules, we have the following results:

$$
\begin{aligned}
& \operatorname{dim}\left(\mathrm{H}^{3}(\mathcal{W}, \mathbb{K})\right)=1 \quad \text { and } \quad \mathrm{H}^{3}(\mathcal{W}, \mathcal{W})=\{0\}, \\
& \operatorname{dim}\left(\mathrm{H}^{3}(\mathcal{V}, \mathbb{K})\right)=1 \quad \text { and } \quad \operatorname{dim}\left(\mathrm{H}^{3}(\mathcal{V}, \mathcal{V})\right)=1 .
\end{aligned}
$$

These results constitute the main part of the present article, given by sections 4 and 5 Moreover, for $\mathrm{H}^{3}(\mathcal{W}, \mathbb{K})$ and $\mathrm{H}^{3}(\mathcal{V}, \mathbb{K})$, we will provide explicit algebraic expressions for the cocycles generating these spaces.

3.4. The Hochschild-Serre Spectral Sequence. In the present article, we will use the Hochschild-Serre spectral sequence. The following theorem is a well-known result in algebraic cohomology:

Theorem [Hochschild-Serre] 3.1. For every ideal $\mathfrak{h}$ of a Lie algebra $\mathfrak{g}$, there is a convergent first quadrant spectral sequence:

$$
\left.E_{2}^{p q}=\mathrm{H}^{p}\left(\mathfrak{g} / \mathfrak{h}, \mathrm{H}^{q}(\mathfrak{h}, M)\right)\right) \Rightarrow \mathrm{H}^{p+q}(\mathfrak{g}, M),
$$

with $M$ being a $\mathfrak{g}$-module and via $\mathfrak{h} \hookrightarrow \mathfrak{g}$ also a $\mathfrak{h}$-module.

A concise proof of this well-known result can for example be found in the textbook by Weibel [31. The original literature is given by the articles [19, 20] by Hochschild and Serre. For knowledge of general spectral sequences, the reader may consult the textbook by McCleary [22].

\section{Analysis OF $\mathrm{H}^{3}(\mathcal{W}, \mathcal{W})$}

In this section, we analyze the third algebraic cohomology of the Witt algebra with values in the adjoint module. We suggest the reader unfamiliar with our techniques to first read the proof in the appendix, which serves as a gently introduction to our methods.

Theorem 4.1. The third algebraic cohomology of the Witt algebra $\mathcal{W}$ over a field $\mathbb{K}$ with char $(\mathbb{K})=0$ and values in the adjoint module vanishes, i.e.

$$
\mathrm{H}^{3}(\mathcal{W}, \mathcal{W})=\{0\} .
$$

Due to the result by Fuks (3.2), we already know that the non-zero degree cohomology of the Witt algebra is zero. However, for reasons of completeness, we will prove this result again for the third cohomology, since the proof is short and simple. Thus, we proceed in two steps, the first step concentrating on the non-zero degree cohomology of the Witt algebra, the second step focusing on the degree zero part.

The condition for a 3 -cochain $\psi$ to be a 3 -cocycle is given by:

$$
\begin{aligned}
& \left(\delta_{3} \psi\right)\left(x_{1}, x_{2}, x_{3}, x_{4}\right) \\
= & \psi\left(\left[x_{1}, x_{2}\right], x_{3}, x_{4}\right)-\psi\left(\left[x_{1}, x_{3}\right], x_{2}, x_{4}\right)+\psi\left(\left[x_{1}, x_{4}\right], x_{2}, x_{3}\right) \\
& +\psi\left(\left[x_{2}, x_{3}\right], x_{1}, x_{4}\right)-\psi\left(\left[x_{2}, x_{4}\right], x_{1}, x_{3}\right)+\psi\left(\left[x_{3}, x_{4}\right], x_{1}, x_{2}\right) \\
& -\left[x_{1}, \psi\left(x_{2}, x_{3}, x_{4}\right)\right]+\left[x_{2}, \psi\left(x_{1}, x_{3}, x_{4}\right)\right]-\left[x_{3}, \psi\left(x_{1}, x_{2}, x_{4}\right)\right]+\left[x_{4}, \psi\left(x_{1}, x_{2}, x_{3}\right)\right]=0,
\end{aligned}
$$


with $x_{1}, x_{2}, x_{3}, x_{4} \in \mathcal{W}$.

The condition for a 3 -cocycle $\psi \in \mathrm{H}^{3}(\mathcal{W}, \mathcal{W})$ to be a coboundary is given by:

$$
\begin{aligned}
\psi\left(x_{1}, x_{2}, x_{3}\right)=\left(\delta_{2} \phi\right)\left(x_{1}, x_{2}, x_{3}\right)= & \phi\left(\left[x_{1}, x_{2}\right], x_{3}\right)+\phi\left(\left[x_{2}, x_{3}\right], x_{1}\right)+\phi\left(\left[x_{3}, x_{1}\right], x_{2}\right) \\
& -\left[x_{1}, \phi\left(x_{2}, x_{3}\right)\right]+\left[x_{2}, \phi\left(x_{1}, x_{3}\right)\right]-\left[x_{3}, \phi\left(x_{1}, x_{2}\right)\right],
\end{aligned}
$$

where $x_{1}, x_{2}, x_{3} \in \mathcal{W}$ and $\phi \in \mathrm{C}^{2}(\mathcal{W}, \mathcal{W})$.

\subsection{The non-zero degree cohomology for the Witt algebra.}

Proposition 4.1. The following holds:

$$
\mathrm{H}_{(d)}^{3}(\mathcal{W}, \mathcal{W})=\{0\} \text { for } d \neq 0 \quad \text { and } \quad \mathrm{H}^{3}(\mathcal{W}, \mathcal{W})=\mathrm{H}_{(0)}^{3}(\mathcal{W}, \mathcal{W}) .
$$

Proof. Let $\psi \in \mathrm{H}_{(d \neq 0)}^{3}(\mathcal{W}, \mathcal{W})$. Let us perform a cohomological change $\psi^{\prime}=\psi-\delta_{2} \phi$ with the following 2-cochain $\phi$ :

$$
\phi\left(x_{1}, x_{2}\right)=-\frac{1}{d} \psi\left(x_{1}, x_{2}, e_{0}\right),
$$

which gives us, taking into account that $\phi\left(e_{0}, \cdot\right)=\phi\left(\cdot, e_{0}\right)=0$ :

$$
\begin{aligned}
& \psi^{\prime}\left(x_{1}, x_{2}, e_{0}\right)=\psi\left(x_{1}, x_{2}, e_{0}\right)-\left(\delta_{2} \phi\right)\left(x_{1}, x_{2}, e_{0}\right) \\
& =\psi\left(x_{1}, x_{2}, e_{0}\right)-\underbrace{\phi\left(\left[x_{1}, x_{2}\right], e_{0}\right)}_{=0}-\phi\left(\left[x_{2}, e_{0}\right], x_{1}\right)-\phi\left(\left[e_{0}, x_{1}\right], x_{2}\right) \\
& +[x_{1}, \underbrace{\phi\left(x_{2}, e_{0}\right)}_{=0}]-[x_{2}, \underbrace{\phi\left(x_{1}, e_{0}\right)}_{=0}]+\left[e_{0}, \phi\left(x_{1}, x_{2}\right)\right] \\
& =\psi\left(x_{1}, x_{2}, e_{0}\right)+\operatorname{deg}\left(x_{2}\right) \underbrace{\phi\left(x_{2}, x_{1}\right)}_{=-\phi\left(x_{1}, x_{2}\right)}-\operatorname{deg}\left(x_{1}\right) \phi\left(x_{1}, x_{2}\right)+\left(\operatorname{deg}\left(x_{1}\right)+\operatorname{deg}\left(x_{2}\right)+d\right) \phi\left(x_{1}, x_{2}\right) \\
& =-d \phi\left(x_{1}, x_{2}\right)+d \phi\left(x_{1}, x_{2}\right)=0 .
\end{aligned}
$$

We thus have $\psi^{\prime}\left(x_{1}, x_{2}, e_{0}\right)=0$. Next, let us write down the cocycle condition for $\psi^{\prime}$ on the quadruplet $\left(x_{1}, x_{2}, x_{3}, e_{0}\right)$ of homogeneous elements:

$$
\begin{aligned}
& \left(\delta_{3} \psi^{\prime}\right)\left(x_{1}, x_{2}, x_{3}, e_{0}\right)=0 \\
\Leftrightarrow & \underbrace{\psi^{\prime}\left(\left[x_{1}, x_{2}\right], x_{3}, e_{0}\right)}_{=0}-\underbrace{\psi^{\prime}\left(\left[x_{1}, x_{3}\right], x_{2}, e_{0}\right)}_{=0}+\psi^{\prime}\left(\left[x_{1}, e_{0}\right], x_{2}, x_{3}\right) \\
& +\underbrace{\psi^{\prime}\left(\left[x_{2}, x_{3}\right], x_{1}, e_{0}\right)}_{=0}-\psi^{\prime}\left(\left[x_{2}, e_{0}\right], x_{1}, x_{3}\right)+\psi^{\prime}\left(\left[x_{3}, e_{0}\right], x_{1}, x_{2}\right) \\
& -[x_{1}, \underbrace{\psi^{\prime}\left(x_{2}, x_{3}, e_{0}\right)}_{=0}]+[x_{2}, \underbrace{\psi^{\prime}\left(x_{1}, x_{3}, e_{0}\right)}_{=0}]-[x_{3}, \underbrace{\psi^{\prime}\left(x_{1}, x_{2}, e_{0}\right)}_{=0}]+\left[e_{0}, \psi^{\prime}\left(x_{1}, x_{2}, x_{3}\right)\right]=0 \\
\Leftrightarrow & -\operatorname{deg}\left(x_{1}\right) \psi\left(x_{1}, x_{2}, x_{3}\right)+\operatorname{deg}\left(x_{2}\right) \underbrace{\psi\left(x_{2}, x_{1}, x_{3}\right)}_{=-\psi\left(x_{1}, x_{2}, x_{3}\right)}-\operatorname{deg}\left(x_{3}\right) \underbrace{\psi\left(x_{3}, x_{1}, x_{2}\right)}_{=\psi\left(x_{1}, x_{2}, x_{3}\right)} \\
& +\left(\operatorname{deg}\left(x_{1}\right)+\operatorname{deg}\left(x_{2}\right)+\operatorname{deg}\left(x_{3}\right)+d\right) \psi\left(x_{1}, x_{2}, x_{3}\right)=0 \\
\Leftrightarrow & d \psi\left(x_{1}, x_{2}, x_{3}\right)=0 \Leftrightarrow \psi\left(x_{1}, x_{2}, x_{3}\right)=0 \text { as } d \neq 0 .
\end{aligned}
$$

We conclude that the third cohomology of the Witt algebra reduces to the degree zero cohomology, in agreement with the result of Fuks (3.2). 
4.2. The degree zero cohomology for the Witt algebra. The proposition we shall prove in this section is the following:

Proposition 4.2. The following holds:

$$
\mathrm{H}_{(0)}^{3}(\mathcal{W}, \mathcal{W})=\{0\}
$$

Clearly, Proposition 4.2 together with Proposition 4.1 shows Theorem 4.1. The proof of Proposition 4.2 is accomplished in six steps and is similar to the proof performed for $\mathrm{H}_{(0)}^{2}(\mathcal{W}, \mathcal{W})$ in $[25,26]$.

Let $\psi$ be a degree zero 3 -cocycle, i.e. we can write it as $\psi\left(e_{i}, e_{j}, e_{k}\right)=\psi_{i, j, k} e_{i+j+k}$ with suitable coefficients $\psi_{i, j, k} \in \mathbb{K}$. We say that $\psi_{,,, .}$is of level $l \in \mathbb{Z}$ if one of its indices is equal to $l$, i.e. $\psi_{,,,,}=\psi_{,,, l}$ or some permutation thereof.

Consequently, five steps of the proof correspond to the analysis of the levels plus one, minus one, zero, plus two and minus two. The final step consists in the analysis of generic levels, which is obtained by induction. In each step, there are always three cases to consider depending on the signs of the indices. One of the three indices corresponds to the level and is fixed. In that case, the three cases to consider correspond to both remaining indices being negative, both being positive, or one being negative and one being positive. It does not matter which of the indices are chosen to be positive or negative, nor does it matter which one of the three indices is chosen to be fixed, because of the alternating property of the cochains. In the following, we provide a brief and superficial summary of the proof:

- Level plus one / minus one: There is a cohomological change $\psi^{\prime}=\psi-\delta_{2} \phi$, $\phi \in C_{(0)}^{2}(\mathcal{W}, \mathcal{W})$ which allows to normalize to zero either the coefficients of level plus one or the coefficients of level minus one, depending on the signs of the two remaining indices. More precisely, we normalize $\psi^{\prime}$ to $\psi_{i, j,-1}^{\prime}=0$ if $i$ and $j$ are both positive and $\psi_{i, j, 1}^{\prime}=0$ else.

The aim is to use the coboundary condition to produce recurrence relations which provide a consistent definition of $\phi$, i.e. of all the $\phi_{i, j} \forall i, j \in \mathbb{Z}$. Each degree of freedom given by some $\phi_{\text {., }}$, should be used to cancel some coefficient of the form $\psi_{,,, 1}$ or $\psi_{,,,-1}$. In the case where both indices of $\phi_{i, j}$ have the same sign, the definition of the $\phi_{i, j}$ 's can be obtained in a straightforward manner from the recurrence relations. In the case where the two indices are of opposite sign, poles occur in the recurrence relations, and the definition of the $\phi_{i, j}$ 's has to be obtained in a somewhat roundabout manner.

- Level zero: For a cocycle $\psi$ normalized as described in the previous bullet point, the cocycle conditions imply $\psi_{i, j, 0}=0 \forall i, j \in \mathbb{Z}$.

The cocycle conditions provide recurrence relations which allow to deduce the result immediately for $i$ and $j$ of the same sign. For $i$ and $j$ of different sign, the proof is an (almost) straightforward generalization of the proof of $\mathrm{H}^{2}(\mathcal{W}, \mathcal{W})_{(0)}=\{0\}$ given in $[25,26]$.

- Level minus one / plus one: The cocycle conditions imply $\psi_{i, j, 1}=0$ if $i$ and $j$ are both positive and $\psi_{i, j,-1}=0$ else. Together with the result of the first bullet point, we have $\psi_{i, j, 1}=\psi_{i, j,-1}=0 \forall i, j \in \mathbb{Z}$.

This step is the simplest one of the entire proof. The cocycle conditions provide again recurrence relations which allow to deduce the results directly. 
- Levels plus two and minus two / Generic Level $k$ : The cocycle conditions imply $\psi_{i, j,-2}=0$ and $\psi_{i, j, 2}=0 \forall i, j \in \mathbb{Z}$. Induction on $k$ subsequently implies $\psi_{i, j, k}=0 \forall i, j, k \in \mathbb{Z}$.

For both indices $i$ and $j$ negative, the first step consists in proving that level minus two is zero, i.e. $\psi_{i, j,-2}=0$. Induction on the third index allows to conclude that the coefficients $\psi_{i, j, k}$ are zero for all negative indices $i, j, k \leq 0$. These results can be obtained directly from the recurrence relations given by the cocycle conditions.

In the case of one positive and one negative index, the first step consists in proving that both levels plus two and minus two are zero, $\psi_{i, j, 2}=\psi_{i, j,-2}=0$. This has to be done by using induction on either $i$ or $j$ depending on the level under consideration. Note that in the proof of $\mathrm{H}^{2}(\mathcal{W}, \mathcal{W})_{(0)}=\{0\}$ in [25, 26], the vanishing of the levels plus two and minus two could be proved directly without using induction. Obviously, the number of times induction has to be used increases with the number of indices. Due to poles and zeros in the recurrence relations, the proof again follows a somewhat roundabout way. The second and final step consists in using induction on the third index in order to prove $\psi_{i, j, k}=0$ for mixed indices, i.e. two indices positive and one index negative or two indices negative and one index positive.

The final case with both indices $i$ and $j$ positive starts with the proof that level plus two is zero, i.e. $\psi_{i, j, 2}=0$. Induction on the third index allows to conclude that the coefficients $\psi_{i, j, k}$ are zero for all positive indices $i, j, k \geq 0$. These results follow directly from the recurrence relations.

We now come to the detailed proof. Let us write down the coboundary and cocycle conditions for later use. If $\phi$ is a degree zero 2-cochain, i.e. $\phi\left(e_{i}, e_{j}\right)=\phi_{i, j} e_{i+j}$, the coboundary condition for $\psi$ on the triplet $\left(e_{i}, e_{j}, e_{k}\right)$ becomes:

$$
\begin{aligned}
\psi_{i, j, k}=\left(\delta_{2} \phi\right)_{i, j, k}= & (j-i) \phi_{i+j, k}+(k-j) \phi_{k+j, i}+(i-k) \phi_{i+k, j} \\
& -(j+k-i) \phi_{j, k}+(i+k-j) \phi_{i, k}-(i+j-k) \phi_{i, j} .
\end{aligned}
$$

The cocycle condition for $\psi$ on the quadruplet $\left(e_{i}, e_{j}, e_{k}, e_{l}\right)$ becomes:

$$
\begin{aligned}
\left(\delta_{3} \psi\right)_{i, j, k, l}= & (j-i) \psi_{i+j, k, l}-(k-i) \psi_{i+k, j, l}+(l-i) \psi_{i+l, j, k} \\
& +(k-j) \psi_{k+j, i, l}-(l-j) \psi_{l+j, i, k}+(l-k) \psi_{l+k, i, j} \\
& -(j+k+l-i) \psi_{j, k, l}+(i+k+l-j) \psi_{i, k, l} \\
& -(i+j+l-k) \psi_{i, j, l}+(i+j+k-l) \psi_{i, j, k}=0 .
\end{aligned}
$$

The first step of the proof is achieved with a cohomological change:

Lemma 4.1. Every 3-cocycle $\psi$ of degree zero is cohomologous to a degree zero 3-cocycle $\psi^{\prime}$ with:

$$
\begin{array}{lll} 
& \psi_{i, j, 1}^{\prime}=0 & \forall i \leq 0, \forall j \in \mathbb{Z}, \\
\text { and } \quad \psi_{i, j,-1}^{\prime}=0 & \forall i, j>0, \\
\text { and } \quad \psi_{i,-1,2}^{\prime}=0 & \forall i \in \mathbb{Z}, \\
\text { and } & \psi_{-4,2,-2}^{\prime}=0 . &
\end{array}
$$

Proof. The aim is to define consistently a 2-cochain $\phi$ that leads to the results (4.1) after the cohomological change. Writing $\phi$ in terms of its coefficients, we start by defining $\phi_{i, 1}=$ $0 \forall i \in \mathbb{Z}$ and $\phi_{-1,2}=0$. Hence, we will perform a cohomological change $\psi^{\prime}=\psi-\delta_{2} \phi$ with $\phi$ 
normalized to $\phi_{i, 1}=0 \forall i \in \mathbb{Z}$ and $\phi_{-1,2}=0$. This simplifies the notations considerably. To increase the readability of the proof, we will separate the analysis depending on the signs of the indices $i, j$. Let us start with the case $i$ and $j$ both being negative.

\section{Case 1: $i, j \leq 0$}

Our aim is to show that we can find coefficients $\phi_{i, j}$ such that $\psi_{i, j, 1}^{\prime}=0$. Writing down the coboundary condition for $(i, j, 1)$ and dropping the terms of the form $\phi_{., 1}$, we need:

$$
\psi_{i, j, 1}=-(i+j-1) \phi_{i, j}+(i-1) \phi_{i+1, j}-(j-1) \phi_{j+1, i} .
$$

This is the case if we define $\phi$ :

$$
\phi_{i, j}:=\frac{i-1}{i+j-1} \phi_{i+1, j}-\frac{j-1}{i+j-1} \phi_{j+1, i}-\frac{\psi_{i, j, 1}}{i+j-1} .
$$

Starting with $i=0, j=-1, j$ decreasing and using $\phi_{., 1}=0$, this recurrence relation defines in a first step $\phi_{0, j}$ for $j \leq-1$. In a second step, $\phi_{-1, j}$ with $j \leq-2$ can be obtained, and so on for all $i \leq-2$ with $j<i$. It is sufficient to consider $j<i$ due to the alternating character of the cochains. Thus, this recurrence relation defines $\phi_{i, j}$ for $i, j \leq 0$. It follows that we can perform a cohomological change such that $\psi_{i, j, 1}^{\prime}=0 \forall i, j \leq 0$.

\section{Case 2: $i \leq 0$ and $j>0$}

We will start by proving that we can obtain $\psi_{i, 2,-1}^{\prime}=0 \forall i \leq 0$ for a suitable choice of the coefficients $\phi_{i, j}$.

Let us consider the coboundary condition for $(-3,2,-1)$. Taking into account the normalization $\phi_{2,-1}=0$ we obtain:

$$
-2 \phi_{-4,2}-6 \phi_{-3,-1}=\psi_{-3,2,-1} .
$$

The quantity $\phi_{-3,-1}$ has been defined in the previous case $i, j \leq 0$. Thus, we obtain a definition for $\phi_{-4,2}$. From there, we can obtain $\phi_{i, 2} i \leq-5$ by using the coboundary condition for $(i, 2,-1)$ and $\phi_{2,-1}=0$, which gives us:

$$
\phi_{i-1,2}=\frac{3+i}{i+1} \phi_{i, 2}+\frac{\psi_{i, 2,-1}}{i+1}-\frac{i-3}{i+1} \phi_{i,-1}+\frac{i-2}{i+1} \phi_{i+2,-1} .
$$

The last two terms have been defined in the previous case $i, j \leq 0$. Thus, this defines $\phi_{i, 2} i \leq-4$ such that we have $\psi_{i, 2,-1}^{\prime}=0 \forall i \leq-3$. Next, let us consider the coboundary condition for $(-4,2,-2)$ :

$$
\psi_{-4,2,-2}=-2 \phi_{-6,2}-8 \phi_{-4,-2}-4 \phi_{0,-4}-4 \phi_{2,-2} .
$$

The coefficients $\phi_{-4,-2}$ and $\phi_{0,-4}$ have been defined in the previous case $i, j \leq 0$. The coefficient $\phi_{-6,2}$ has been defined in $(4.2)$ for $i \leq-4$. Therefore, we obtain a definition for $\phi_{2,-2}$, which annihilates $\psi_{-4,2,-2}^{\prime}, \psi_{-4,2,-2}^{\prime}=0$.

As $\phi_{2,-2}$ is now defined, we can come back to Equation 4.2 , insert $i=-2$ and obtain a definition for $\phi_{-3,2}$, annihilating $\psi_{-2,2,-1}^{\prime}$. Since $\phi_{-1,2}=0$ due to our normalization, the only remaining $\phi_{i, 2} i \leq 0$ to define is $\phi_{0,2}$.

Let us write down the coboundary condition for $(0,2,-1)$ :

$$
\begin{gathered}
-\left(3 \phi_{0,2}+3 \phi_{0,-1}\right)=\psi_{0,2,-1} \\
\Leftrightarrow \phi_{0,2}=-\phi_{0,-1}-\frac{1}{3} \psi_{0,2,-1} .
\end{gathered}
$$


This defines $\phi_{0,2}$ and consequently, $\psi_{0,2,-1}^{\prime}=0$. Since $\psi_{-1,2,-1}^{\prime}=0$ due to the alternating property, we obtain all in all that $\psi_{i, 2,-1}^{\prime}=0 \forall i \leq 0$.

Next, let us prove that we can obtain $\psi_{i, j, 1}^{\prime}=0 \forall i \leq 0 \forall j>0$. It suffices to write down the coboundary condition for $(i, j, 1)$ in the following way:

$$
\phi_{i, j+1}:=\frac{i+j-1}{j-1} \phi_{i, j}-\frac{i-1}{j-1} \phi_{i+1, j}+\frac{\psi_{i, j, 1}}{j-1} .
$$

Fixing $i=0$, and starting with $j=2$ (recall that $\phi_{i, 1}=0$ and that we have just defined all $\left.\phi_{i, 2} i \leq 0\right), j$ increasing, we obtain $\phi_{0, j} \forall j>2$ and $\psi_{0, j, 1}^{\prime}=0 \forall j \geq 2$. Similarly, fixing $i=-1$, and starting with $j=2, j$ increasing, we obtain $\phi_{-1, j} \forall j>2$ and $\psi_{-1, j, 1}^{\prime}=0 \forall j \geq 2$. Continuing along the same lines, we obtain $\phi_{i, j} \forall i \leq 0, j>0$ and $\psi_{i, j, 1}^{\prime}=0 \forall i \leq 0, j>0$. Together with the result $\psi_{i, j, 1}^{\prime}=0 \forall i, j \leq 0$ obtained from the previous case with $i, j \leq 0$, we get $\psi_{i, j, 1}^{\prime}=0 \forall i \leq 0, \forall j \in \mathbb{Z}$

Case 3: $i>0$ and $j>0$

Let us write down the coboundary condition for $(i, j,-1)$ :

$$
\begin{aligned}
\psi_{i, j,-1}= & (i+1) \phi_{i-1, j}+(i-j-1) \phi_{i,-1}-(1+i+j) \phi_{i, j} \\
& +(j+1) \phi_{i, j-1}+(1+i-j) \phi_{j,-1}+(j-i) \phi_{i+j,-1} .
\end{aligned}
$$

From there, we can define $\phi$ via recurrence as follows:

$$
\begin{aligned}
\phi_{i, j}= & \frac{(i+1)}{(1+i+j)} \phi_{i-1, j}+\frac{(j+1)}{(1+i+j)} \phi_{i, j-1}-\frac{\psi_{i, j,-1}}{(1+i+j)} \\
& +\frac{(i-j-1)}{(1+i+j)} \phi_{i,-1}+\frac{(1+i-j)}{(1+i+j)} \phi_{j,-1}+\frac{(j-i)}{(1+i+j)} \phi_{i+j,-1} .
\end{aligned}
$$

Note that $\phi_{.,-1}$ have been defined in the previous case for $i \leq 0, j>0$. Starting with $i=2$, $j=3$ and $j$ increasing, we obtain in a first step $\phi_{2, j}, \forall j \geq 3$ and $\psi_{2, j,-1}^{\prime}=0 \forall j \geq 3$. Next, fixing $i=3$, starting with $j=4$ and $j$ increasing, we obtain in a second step $\phi_{3, j}, \forall j \geq 4$ and $\psi_{3, j,-1}^{\prime}=0 \forall j \geq 4$. Continuing similarly with $i$ increasing, we finally obtain all $\phi_{i, j}, \forall i, j>0$, and $\psi_{i, j,-1}^{\prime}=0 \forall i, j>0$. Note that we already have $\psi_{1, j,-1}^{\prime}=0 \forall j>0$ due to the previous case, which yielded $\psi_{i, j, 1}^{\prime}=0 \forall i \leq 0, \forall j \in \mathbb{Z}$. Combining the result $\psi_{i, j,-1}^{\prime}=0 \forall i, j>0$ with the result $\psi_{i, 2,-1}^{\prime}=0 \forall i \leq 0$ from the previous case $i \leq 0, j>0$, we also obtain $\psi_{i, 2,-1}^{\prime}=0 \forall i \in \mathbb{Z}$.

Lemma 4.2. Let $\psi$ be a degree zero 3-cocycle such that:

$$
\begin{array}{ll} 
& \psi_{i, j, 1}=0 \quad \forall i \leq 0, \forall j \in \mathbb{Z}, \\
\text { and } \quad \psi_{i, j,-1}=0 \quad \forall i, j>0, \\
\text { and } \quad \psi_{i,-1,2}=0 \quad \forall i \in \mathbb{Z},
\end{array}
$$

then

$$
\psi_{i, j, 0}=0 \quad \forall i, j \in \mathbb{Z} .
$$

Proof. Again, we split the proof into the three cases depending on the signs of $i$ and $j$.

Case 1: $i, j \leq 0$ 
Let us write down the cocycle condition for $(i, j, 0,1)$, neglecting the terms of the form $\psi_{i, j, 1} i, j \leq 0$ :

$$
(i+j-1) \psi_{i, j, 0}-(i-1) \psi_{i+1, j, 0}+(j-1) \psi_{j+1, i, 0}=0 .
$$

We can define the following recurrence relation for $i$ and $j$ decreasing:

$$
\psi_{i, j, 0}=\frac{(i-1)}{(i+j-1)} \psi_{i+1, j, 0}-\frac{(j-1)}{(i+j-1)} \psi_{j+1, i, 0} .
$$

Fixing $i=-1$, starting with $j=-2$ and $j$ decreasing, we obtain $\psi_{-1, j, 0}=0 \forall j \leq-2$. Repeating the same procedure with decreasing values for $i$ and $j<i$, we obtain $\psi_{i, j, 0}=$ $0 \forall i, j \leq 0$.

Case 2: $i \leq 0, j>0$

Let us write down the cocycle condition for $(i, 2,0,-1)$ :

$$
\begin{aligned}
& -\psi_{1, i, 2}+3 \psi_{1, i, 0}+(-1+i) \psi_{2,0,-1}-2 \psi_{2, i,-1}-(1+i) \psi_{-1+i, 2,0} \\
& +(-3+i) \psi_{i, 0,-1}-\psi_{i, 2,-1}+(3+i) \psi_{i, 2,0}-(-2+i) \psi_{2+i, 0,-1}=0 .
\end{aligned}
$$

The slashed terms cancel each other, although they are zero anyway as we have $\psi_{i, 2,-1}=$ $0 \forall i \in \mathbb{Z}$. The term $\psi_{1, i, 0}$ is zero as we have $\psi_{i, j, 1}=0 \forall i, j \leq 0$. The term $\psi_{2,0,-1}$ is zero due to $\psi_{i, 2,-1}=0 \forall i \in \mathbb{Z}$. The terms $\psi_{i, 0,-1}$ and $\psi_{2+i, 0,-1}$ (for $i \leq-2$ ) are zero because of Case 1, i.e. they are of the form $\psi_{i, j, 0}=0 \forall i, j \leq 0$. Therefore, we are left with:

$$
\psi_{i-1,2,0}=\frac{i+3}{i+1} \psi_{i, 2,0} .
$$

Putting $i=-3$ in the equation above, this recurrence relation implies $\psi_{-4,2,0}=0$ and by recursion $\psi_{i, 2,0}=0 \forall i \leq-4$. Next, consider the cocycle condition for $(i, 2,-2,0)$ :

$$
\begin{aligned}
& 2 \psi_{-2, i, 2}+i \psi_{2,-2,0}+2 \psi_{2, i,-2}+(2+i) \psi_{-2+i, 2,0} \\
& +(-4+i) \psi_{i,-2,0}-(4+i) \psi_{i, 2,0}-(-2+i) \psi_{2+i,-2,0}=0 .
\end{aligned}
$$

The slashed terms cancel each other, the terms $\psi_{i,-2,0}$ and $\psi_{2+i,-2,0}$ (for $i \leq-2$ ) are zero because of $\psi_{i, j, 0}=0 \forall i, j \leq 0$. As we have $\psi_{i, 2,0}=0 \forall i \leq-4$, we can put for example $i=-4$ in the equation above and obtain $\psi_{2,-2,0}=0$. Inserting this value in Equation (4.4) with $i=-2$, we obtain $\psi_{-3,2,0}=0$. Recall that we also have $\psi_{-1,2,0}=0$ due to $\psi_{i,-1,2}=0 \forall i \in \mathbb{Z}$. All in all, we have $\psi_{i, 2,0} \forall i \leq 0$.

This result is needed to write down a well-defined recurrence relation. Writing down the cocycle condition for $(i, j, 0,1)$ and neglecting the terms of the form $\psi_{i, j, 1}$ with $i, j \leq 0$ and $i \leq 0, j>0$, we obtain the following recurrence relation:

$$
\psi_{i, j+1,0}=\frac{i+j-1}{j-1} \psi_{i, j, 0}-\frac{i-1}{j-1} \psi_{i+1, j, 0} .
$$

Fixing $i=-1$, one starts with $j=2$ (since we already have $\psi_{i, j, 1}=0 i, j \leq 0$ and $\psi_{i, 2,0}=$ $0 i \leq 0$ ), which gives, with increasing $j, \psi_{-1, j, 0}=0 j \geq 3$. Continuing with fixing $i=-2$, starting again with $j=2$ and increasing $j$, we obtain $\psi_{-2, j, 0}=0 j \geq 3$. Doing this for all $i \leq 0$, we finally obtain $\psi_{i, j, 0}=0 \forall i \leq 0, j>0$.

Case 3: $i, j>0$ 
Writing down the cocycle condition for $(i, j, 0,-1)$, we obtain:

$$
\begin{aligned}
& -\psi_{-1, i, j}-(1+i) \psi_{-1+i, j, 0}+(-1+i-j) \psi_{i, 0,-1}+i \psi_{i, j,-1}-(-1+i+j) \psi_{i, j,-1} \\
& +(1+i+j) \psi_{i, j, 0}+(1+j) \psi_{-1+j, i, 0}+(1+i-j) \psi_{j, 0,-1}-j \psi_{j, i,-1}+(-i+j) \psi_{i+j, 0,-1}=0 .
\end{aligned}
$$

The slashed terms cancel each other, though they are zero anyway due to $\psi_{i, j,-1}=0 i, j>0$. The terms $\psi_{i, 0,-1}, \psi_{j, 0,-1}$ and $\psi_{i+j, 0,-1}$ are zero due to the previous case, $\psi_{i, j, 0}=0 i \leq 0, j>$ 0 . Thus, we obtain the following recurrence relation:

$$
\psi_{i, j, 0}=\frac{(1+i)}{(1+i+j)} \psi_{-1+i, j, 0}-\frac{(1+j)}{(1+i+j)} \psi_{-1+j, i, 0} .
$$

Fixing $i=1$, starting with $j=2, j$ increasing, we obtain $\psi_{1, j, 0}=0 j \geq 2$. Fixing $i=2$, starting with $j=3$, we get $\psi_{2, j, 0}=0 j \geq 3$. Continuing with increasing $i$ and keeping $j>i$ due to skew-symmetry, we finally obtain $\psi_{i, j, 0}=0 \forall i, j>0$.

Taking all three cases together, we obtain the announced result, $\psi_{i, j, 0}=0 \forall i, j \in \mathbb{Z}$.

Lemma 4.3. Let $\psi$ be a degree zero 3-cocycle such that:

$$
\begin{array}{lll} 
& \psi_{i, j, 1}=0 & \forall i \leq 0, \forall j \in \mathbb{Z}, \\
\text { and } & \psi_{i, j,-1}=0 & \forall i, j>0, \\
\text { and } & \psi_{i,-1,2}=0 & \forall i \in \mathbb{Z}, \\
\text { and } & \psi_{i, j, 0}=0 & \forall i, j \in \mathbb{Z},
\end{array}
$$

then

$$
\psi_{i, j, 1}=\psi_{i, j,-1}=0 \quad \forall i, j \in \mathbb{Z} .
$$

Proof. Again, the proof is split into the three cases depending on the signs of $i, j$.

\section{Case 1: $i, j \leq 0$}

Writing down the cocycle condition for $(i, j, 1,-1)$ and neglecting $\psi_{i, j, 1} i, j \leq 0$ as well as $\psi_{i, j, 0} i, j \leq 0$, we obtain:

$$
\begin{aligned}
& -(-2+i+j) \psi_{i, j,-1}+(-1+i) \psi_{1+i, j,-1}-(-1+j) \psi_{1+j, i,-1}=0 \\
\Leftrightarrow & \psi_{i, j,-1}=\frac{(-1+i)}{(-2+i+j)} \psi_{1+i, j,-1}-\frac{(-1+j)}{(-2+i+j)} \psi_{1+j, i,-1} .
\end{aligned}
$$

Fixing $i=-2$ (since level zero $\psi_{0, j,-1} j \leq 0$ is already done and $\psi_{-1, j,-1}=0$ ), starting with $j=-3$ and $j$ decreasing, we obtain $\psi_{-2, j,-1}=0 j \leq-3$. Fixing $i=-3$, starting with $j=-4$ and $j$ decreasing, we get $\psi_{-3, j,-1}=0 j \leq-4$. Continuing along the same lines, we obtain $\psi_{i, j,-1}=0 i, j \leq 0$.

Case 2: $i \leq 0, j>0$

Writing down the cocycle condition for $(i, j, 1,-1)$ and neglecting $\psi_{i, j, 1} i \leq 0, j>0$, $\psi_{i, j, 1} i, j \leq 0$ as well as $\psi_{i, j, 0} i \leq 0, j>0$, we obtain:

$$
\begin{aligned}
& -(-2+i+j) \psi_{i, j,-1}+(-1+i) \psi_{1+i, j,-1}-(-1+j) \psi_{1+j, i,-1}=0 \\
& \Leftrightarrow \psi_{i, 1+j,-1}=\frac{(-2+i+j)}{(-1+j)} \psi_{i, j,-1}-\frac{(-1+i)}{(-1+j)} \psi_{1+i, j,-1} .
\end{aligned}
$$

Fixing $i=-2$ (since level zero $\psi_{0, j,-1} j>0$ is already done and $\psi_{-1, j,-1}=0$ ) and starting with $j=2$ (since $\psi_{i, 2,-1}=0 i \leq 0$ ), increasing $j$, we obtain $\psi_{-2, j,-1}=0 j \geq 3$. Fixing $i=-3$, starting again with $j=2, j$ increasing, we get $\psi_{-3, j,-1}=0 j \geq 3$. Continuing with 
$i$ decreasing, we get $\psi_{i, j,-1}=0 i \leq 0, j>0$.

Case 3: $i, j>0$

Writing again down the cocycle condition for $(i, j, 1,-1)$, this time neglecting the terms $\psi_{i, j,-1} i, j>0$ and $\psi_{i, j, 0} i, j>0$, we obtain:

$$
\begin{aligned}
& -(1+i) \psi_{-1+i, j, 1}+(2+i+j) \psi_{i, j, 1}+(1+j) \psi_{-1+j, i, 1}=0 \\
& \Leftrightarrow \psi_{i, j, 1}=\frac{(1+i)}{(2+i+j)} \psi_{-1+i, j, 1}-\frac{(1+j)}{(2+i+j)} \psi_{-1+j, i, 1} .
\end{aligned}
$$

Fixing $i=2\left(\right.$ since $\left.\psi_{1, j, 1}=0\right)$ and starting with $j=3$, increasing $j$, we obtain $\psi_{2, j, 1}=0 j \geq$ 3. Increasing $i$ and keeping $j>i$ we finally obtain $\psi_{i, j, 1}=0 \forall i, j>0$.

Taking all three cases together, we have proven that $\psi_{i, j, 1}=0 \forall i, j \in \mathbb{Z}$ and $\psi_{i, j,-1}=$ $0 \forall i, j \in \mathbb{Z}$.

Lemma 4.4. Let $\psi$ be a degree zero 3-cocycle such that:

$$
\psi_{i, j, 1}=\psi_{i, j,-1}=\psi_{i, j, 0}=0 \quad \forall i, j \in \mathbb{Z} \quad \text { and } \quad \psi_{-4,2,-2}=0,
$$

then

$$
\psi_{i, j, k}=0 \quad \forall i, j, k \in \mathbb{Z} .
$$

Proof. Again, the proof is split in the three cases depending on the signs of $i$ and $j$.

Case 1: $i, j \leq 0$

In a first step, we shall prove the following statement: $\psi_{i, j,-2}=0 \forall i, j \leq 0$.

Writing down the cocycle condition for $(i, j,-2,1)$ and neglecting the terms of level one $\psi_{i, j, 1}$ and level minus one $\psi_{i, j,-1}$, we obtain the following:

$$
\begin{aligned}
& (-3+i+j) \psi_{i, j,-2}-(-1+i) \psi_{1+i, j,-2}+(-1+j) \psi_{1+j, i,-2}=0 \\
& \Leftrightarrow \psi_{i, j,-2}=\frac{(-1+i)}{(-3+i+j)} \psi_{1+i, j,-2}-\frac{(-1+j)}{(-3+i+j)} \psi_{1+j, i,-2} .
\end{aligned}
$$

Fixing $i=-3$ (since the levels zero $\psi_{i, j, 0}$ and minus one $\psi_{i, j,-1}$ are already done and $\psi_{-2, j,-2}=0$ ), starting with $j=-4$ and decreasing $j$, we obtain $\psi_{-3, j,-2}=0 j \leq-4$. Continuing along the same lines with decreasing $i$ and keeping $j<i$, we obtain $\psi_{i, j,-2}=$ $0 \forall i, j \leq 0$ as a first step.

In a second step, we shall prove $\psi_{i, j, k}=0 \forall i, j, k \leq 0$. This can be done by induction. We know the result is true for $k=0,-1,-2$. Hence, we will assume it is true for some $k \leq-2$ and check whether it remains true for $k-1$. The cocycle condition for $(i, j, k,-1)$ is given by, after omitting terms of level minus one $\psi_{i, j,-1}$ :

$$
\begin{aligned}
& -(1+i) \psi_{-1+i, j, k}+(1+i+j+k) \psi_{i, j, k}+(1+j) \psi_{-1+j, i, k}-(1+k) \psi_{-1+k, i, j}=0 \\
& \Leftrightarrow-(1+k) \psi_{-1+k, i, j}=0 \Leftrightarrow \psi_{-1+k, i, j}=0 \text { as } k \leq-2 .
\end{aligned}
$$

The terms $\psi_{-1+i, j, k}, \psi_{i, j, k}$ and $\psi_{-1+j, i, k}$ are zero since they are of level $k$ and thus zero by induction hypothesis. It follows $\psi_{i, j, k}=0 \forall i, j, k \leq 0$.

Case 2: $i \leq 0, j>0$

In a first step, we shall prove the following statements: $\psi_{i, j,-2}=0 \forall i \leq 0, j>0$ and $\psi_{i, j, 2}=0 \forall i \leq 0, j>0$. 
The cocycle condition for $(-3,2,-2,-1)$ reads, after dropping the terms of level one, minus one and zero:

$$
2 \psi_{-4,2,-2}-2 \psi_{-3,2,-2}=0 .
$$

Since we have $\psi_{-4,2,-2}=0$, the equation above implies $\psi_{-3,2,-2}=0$. Next, let us write down the cocycle condition for $(-3, j,-2,1)$, which gives after dropping terms of level one and of level minus one:

$$
\begin{aligned}
& (-6+j) \psi_{-3, j,-2}+(-1+j) \psi_{1+j,-3,-2}=0 \\
& \Leftrightarrow \psi_{1+j,-3,-2}=\frac{(-6+j)}{(-1+j)} \psi_{j,-3,-2} .
\end{aligned}
$$

Starting with $j=2$, we obtain $\psi_{j,-3,-2}=0 \forall j \geq 3$ since the starting point is zero: $\psi_{2,-3,-2}=0$. Adding the level one, we obtain $\psi_{j,-3,-2}=0 \forall j>0$.

Next, let us write down the cocycle condition for $(i, 3,2,-1)$ after dropping terms of level one and level minus one:

$$
\begin{aligned}
& -(1+i) \psi_{-1+i, 3,2}+(6+i) \psi_{i, 3,2}=0 \\
& \Leftrightarrow \psi_{-1+i, 3,2}=\frac{(6+i)}{(1+i)} \psi_{i, 3,2} .
\end{aligned}
$$

This gives us for $i=-2$ : $\psi_{-3,3,2}=-4 \psi_{-2,3,2}$.

For $i=-3: \psi_{-4,3,2}=\frac{3}{-2} \psi_{-3,3,2}=6 \psi_{-2,3,2}$.

For $i=-4: \psi_{-5,3,2}=\frac{2}{-3} \psi_{-4,3,2}=-4 \psi_{-2,3,2}$.

Now, let us write down the cocycle condition for $(-3,3,2,-2)$ and drop the terms of level one, level minus one and level zero, as well as the terms of the form $\psi_{j,-3,-2} j>0$ :

$$
\begin{aligned}
& \psi_{-5,3,2}+4 \psi_{-3,3,2}-6 \psi_{3,2,-2}=0 \\
& \Leftrightarrow-4 \psi_{-2,3,2}-16 \psi_{-2,3,2}-6 \psi_{-2,3,2}=0 \\
& \Leftrightarrow \psi_{-2,3,2}=0 .
\end{aligned}
$$

To obtain the second line, the first two terms were simply replaced by their expressions computed above. Putting $i=-2$ and $\psi_{-2,3,2}=0$ in the recurrence relation (4.5), we obtain $\psi_{i, 3,2}=0 \forall i \leq-3$. Together with the levels minus one and zero, we obtain $\psi_{i, 3,2}=0 \forall i \leq 0$. To prove $\psi_{i, j,-2}=0 \forall i \leq 0, j>0$, we will use induction on $i$. Indeed, we have proven that $\psi_{i, j,-2}=0 \forall j>0$, for $i=0,-1,-2,-3$ (recall that we have $\psi_{j,-3,-2}=0 j>0$ ). Suppose the statement holds true down to $i+1, i \leq-4$, and let us see what happens for $i$. The cocycle condition for $(i, j,-2,1)$ gives, after dropping terms of level one and level minus one:

$$
\begin{aligned}
& (-3+i+j) \psi_{i, j,-2}-(-1+i) \underbrace{\psi_{1+i, j,-2}}_{=0}+(-1+j) \psi_{1+j, i,-2}=0 \\
& \Leftrightarrow \psi_{i, j+1,-2}=\frac{(-3+i+j)}{(-1+j)} \psi_{i, j,-2} .
\end{aligned}
$$

The term in the middle is zero due to the induction hypothesis.

This gives, for $j=2: \psi_{i, 3,-2}=(-1+i) \psi_{i, 2,-2}$.

For $j=3: \psi_{i, 4,-2}=\frac{i}{2} \psi_{i, 3,-2}=\frac{(-1+i)(i)}{2} \psi_{i, 2,-2}$.

For $j=4: \psi_{i, 5,-2}=\frac{(1+i)}{3} \psi_{i, 4,-2}=\frac{(-1+i)(i)(1+i)}{6} \psi_{i, 2,-2}$. 
Next, we will insert these values into the cocycle condition for $(i, 3,-2,2)$, after dropping terms of level zero and level one:

$$
\begin{aligned}
& (-3+i) \psi_{3,-2,2}+\psi_{5, i,-2}+(2+i) \psi_{-2+i, 3,2}+(-3+i) \psi_{i,-2,2} \\
& +(-1+i) \psi_{i, 3,-2}-(7+i) \psi_{i, 3,2}-(-2+i) \psi_{2+i, 3,-2}-(-3+i) \psi_{3+i,-2,2}=0 \\
& \Leftrightarrow-\frac{(-1+i)(i)(1+i)}{6} \psi_{i, 2,-2}-(-3+i) \psi_{i, 2,-2}+(-1+i)(-1+i) \psi_{i, 2,-2}=0 \\
& \Leftrightarrow(i-3)\left(i^{2}-3 i+8\right) \psi_{i, 2,-2}=0 .
\end{aligned}
$$

The terms $\psi_{3,-2,2}, \psi_{-2+i, 3,2}$ and $\psi_{i, 3,2}$ are zero due to what was proved before, $\psi_{i, 3,2}=$ $0 \forall i \leq 0$. The terms $\psi_{2+i, 3,-2}$ and $\psi_{3+i,-2,2}$ are zero as a consequence of the induction hypothesis. In the last line, we have $(i-3) \neq 0$, since $i \leq-4$, and also $\left(i^{2}-3 i+8\right) \neq 0$ since its discriminant is negative. It follows $\psi_{i, 2,-2}=0$. Reinserting this into (4.6) and taking into account that level one is zero, we obtain that the induction holds true for $i$, and thus: $\psi_{i, j,-2}=0 \forall i \leq 0, j>0$.

Next, we proceed similarly, but with induction on $j$, to prove $\psi_{i, j, 2}=0 \forall i \leq 0, j>0$. We already know that the statement holds true for $j=1,2,3$. Let us suppose it is true up to $j-1, j \geq 4$, and show that it remains true for $j$. Let us write down the cocycle condition for $(i, j, 2,-1)$, after dropping terms of level one and level minus one:

$$
\begin{aligned}
& -(1+i) \psi_{-1+i, j, 2}+(3+i+j) \psi_{i, j, 2}+(1+j) \underbrace{\psi_{-1+j, i, 2}}_{=0}=0 \\
& \Leftrightarrow \psi_{-1+i, j, 2}=\frac{(3+i+j)}{(1+i)} \psi_{i, j, 2} .
\end{aligned}
$$

The third term is zero due to the induction hypothesis. From the recurrence relation above, we obtain for $i=-2: \psi_{-3, j, 2}=-(1+j) \psi_{-2, j, 2}$.

For $i=-3: \psi_{-4, j, 2}=\frac{j}{(-2)} \psi_{-3, j, 2}=\frac{j(1+j)}{2} \psi_{-2, j, 2}$

For $i=-4: \psi_{-5, j, 2}=\frac{(-1+j)}{(-3)} \psi_{-4, j, 2}=-\frac{(-1+j) j(1+j)}{6} \psi_{-2, j, 2}$.

Next, we insert these values into the cocycle condition for $(-3, j, 2,-2)$ after dropping terms of level zero and level minus one:

$$
\begin{aligned}
& \psi_{-5, j, 2}-(3+j) \psi_{-3,2,-2}-(-7+j) \psi_{-3, j,-2}+(1+j) \psi_{-3, j, 2} \\
& +(3+j) \psi_{-3+j, 2,-2}+(2+j) \psi_{-2+j,-3,2}-(3+j) \psi_{j, 2,-2}-(-2+j) \psi_{2+j,-3,-2}=0 \\
& \Leftrightarrow-\frac{(-1+j) j(1+j)}{6} \psi_{-2, j, 2}-(1+j)(1+j) \psi_{-2, j, 2}-(3+j) \psi_{-2, j, 2}=0 \\
& \Leftrightarrow(j+3)\left(8+3 j+j^{2}\right) \psi_{-2, j, 2}=0 .
\end{aligned}
$$

The terms $\psi_{-3,2,-2}, \psi_{-3, j,-2}$ and $\psi_{2+j,-3,-2}$ are zero due to what was shown before in this proof, $\psi_{j,-3,-2}=0 \forall j>0$. The terms $\psi_{-3+j, 2,-2}$ and $\psi_{-2+j,-3,2}$ are zero due to the induction hypothesis. In the last line, we have $(j+3) \neq 0$, since $j \geq 4$, and also $\left(j^{2}+3 j+8\right) \neq 0$ since its discriminant is negative. It follows $\psi_{-2, j, 2}=0$. Reinserting this into (4.7) and taking into account that level minus one is zero, we obtain that the induction holds true for $j$, and thus: $\psi_{i, j, 2}=0 \forall i \leq 0, j>0$.

Now that the terms of level two and of level minus two are zero for the case $i \leq 0, j>0$, we can use induction on $k$ to first prove $\psi_{i, j, k}=0, \forall i \leq 0, j>0, k \geq 0$ and then 
$\psi_{i, j, k}=0, \forall i \leq 0, j>0, k \leq 0$.

The result is true for $k=0,1,2$. Let us assume the result is true for $k, k \geq 2$ and show that it remains true for $k+1$. The cocycle condition for $(i, j, k, 1)$ gives, after dropping terms of level one,

$$
\begin{aligned}
& (-1+i+j+k) \psi_{i, j, k}-(-1+i) \psi_{1+i, j, k}+(-1+j) \psi_{1+j, i, k}-(-1+k) \psi_{1+k, i, j}=0 \\
& \Leftrightarrow(-1+k) \psi_{1+k, i, j}=0 \Leftrightarrow \psi_{1+k, i, j}=0 \text { as } k \geq 2 .
\end{aligned}
$$

The terms $\psi_{i, j, k}, \psi_{1+i, j, k}$ and $\psi_{1+j, i, k}$ are zero because of the induction hypothesis (if $1+i=1$, the term $\psi_{1+i, j, k}$ is still zero because the level plus one is zero for all $j, k \in \mathbb{Z}$ ). It follows that the result holds true for $k+1$.

All the same, the result is true for $k=0,-1,-2$. Let us assume it is true for $k, k \leq-2$, and show that it holds true for $k-1$. The cocycle condition for $(i, j, k,-1)$ yields, after dropping terms of level minus one,

$$
\begin{aligned}
& -(1+i) \psi_{-1+i, j, k}+(1+i+j+k) \psi_{i, j, k}+(1+j) \psi_{-1+j, i, k}-(1+k) \psi_{-1+k, i, j}=0 \\
& \Leftrightarrow(1+k) \psi_{-1+k, i, j}=0 \Leftrightarrow \psi_{-1+k, i, j}=0 \text { as } k \leq-2 .
\end{aligned}
$$

The terms $\psi_{-1+i, j, k}, \psi_{i, j, k}$ and $\psi_{-1+j, i, k}$ are zero because of the induction hypothesis (if $-1+j=0$, the term $\psi_{-1+j, i, k}$ is still zero because the level zero vanishes for all $i, k \in \mathbb{Z}$ ). It follows that the result holds true for $k-1$. Thus, we have obtained the desired result for the case $i \leq 0, j>0$.

\section{Case 3: $i>0, j>0$}

In a first step, we shall prove the following statement: $\psi_{i, j, 2}=0 \forall i, j>0$. The cocycle condition for $(i, j, 2,-1)$ yields, after dropping the terms of level one and of level minus one:

$$
\begin{aligned}
& -(1+i) \psi_{-1+i, j, 2}+(3+i+j) \psi_{i, j, 2}+(1+j) \psi_{-1+j, i, 2}=0 \\
& \Leftrightarrow \psi_{i, j, 2}=\frac{(1+i)}{(3+i+j)} \psi_{-1+i, j, 2}+\frac{(1+j)}{(3+i+j)} \psi_{i,-1+j, 2} .
\end{aligned}
$$

Fixing $i=3$ and starting with $j=4, j$ ascending, we obtain $\psi_{3, j, 2}=0 \forall j \geq 4$. Fixing $i=4$ and starting with $j=5, j$ ascending, we get $\psi_{4, j, 2}=0 \forall j \geq 5$. Continuing with ascending $i$, keeping $j>i$, we finally obtain $\psi_{i, j, 2}=0 \forall i, j>0$.

Finally, we want to prove $\psi_{i, j, k}=0 \forall i, j>0, k \geq 0$. This can be done with induction on $k$. Indeed, the result is true for level zero, level one and level two, i.e. $k=0,1,2$. Thus, let us assume the result is true for $k, k \geq 2$ and show that it holds true for $k+1$. The cocycle condition for $(i, j, k, 1)$ gives, after dropping the terms of level one:

$$
\begin{aligned}
& (-1+i+j+k) \psi_{i, j, k}-(-1+i) \psi_{1+i, j, k}+(-1+j) \psi_{1+j, i, k}-(-1+k) \psi_{1+k, i, j}=0 \\
& \Leftrightarrow(-1+k) \psi_{1+k, i, j}=0 \Leftrightarrow \psi_{1+k, i, j}=0 \text { as } k \geq 2 .
\end{aligned}
$$

The terms $\psi_{i, j, k}, \psi_{1+i, j, k}$ and $\psi_{1+j, i, k}$ are zero because of the induction hypothesis. It follows that the statement holds true for $k+1$.

Taking all three cases together, we find the announced result $\psi_{i, j, k}=0 \forall i, j, k \in \mathbb{Z}$

Proof of Proposition 4.2. Let us collect the statements of the four lemmata. Let $\psi$ be a degree-zero 3-cocycle of $\mathcal{W}$ with values in $\mathcal{W}$. By Lemma 4.1 we can perform a cohomological change such that we obtain a cohomologous degree-zero 3-cocycle with coefficients 
fulfilling (4.1). Hence, the assumptions of Lemma 4.2 are satisfied and we obtain (4.3). Together with Lemma 4.3, the assumptions of Lemma 4.4 are fulfilled and Lemma 4.4 shows $\psi_{i, j, k}=0 \forall i, j, k \in \mathbb{Z}$, which proves the Proposition 4.2

\section{Analysis OF $\mathrm{H}^{3}(\mathcal{V}, \mathcal{V})$}

The aim of this section is to prove the following result:

Theorem 5.1. The third algebraic cohomology of the Virasoro algebra $\mathcal{V}$ over a field $\mathbb{K}$ with $\operatorname{char}(\mathbb{K})=0$ and values in the adjoint module is one-dimensional, i.e.

$$
\operatorname{dim}\left(\mathrm{H}^{3}(\mathcal{V}, \mathcal{V})\right)=1
$$

Proof. Theorem 5.1 can be shown by direct computations using elementary algebra. However, the resulting proof is rather long and complicated. We prefer to prove this result by using higher tools from cohomological algebra, which has also the advantage to yield interesting intermediate results. Moreover, it exhibits the relations between the various cohomology groups in an efficient way.

The proof is divided into two main steps:

- Theorem 5.2 Proof of $\mathrm{H}^{3}(\mathcal{V}, \mathcal{W}) \cong \mathrm{H}^{3}(\mathcal{W}, \mathcal{W})$.

- Theorem 5.3. Proof of $\operatorname{dim}\left(\mathrm{H}^{3}(\mathcal{V}, \mathbb{K})\right)=\operatorname{dim}\left(\mathrm{H}^{3}(\mathcal{W}, \mathbb{K})\right)=1$.

Theorem 5.2 will be derived via spectral sequences. Theorem 5.3 can be shown by using elementary algebraic manipulations. These theorems will be proven below. For now, we will assume that these results hold and we will proceed with the proof of Theorem 5.1.

The short exact sequence of Lie algebras in 2.1) can also be viewed as a short exact sequence of $\mathcal{V}$-modules. In fact, the Witt algebra $\mathcal{W}$ is a $\mathcal{V}$-module. Furthermore, the base field $\mathbb{K}$ is a trivial $\mathcal{V}$-module. This short exact sequence of $\mathcal{V}$-modules gives rise to a long exact sequence in cohomology, the relevant part for us being the third cohomology:

$$
\cdots \rightarrow \mathrm{H}^{2}(\mathcal{V}, \mathcal{W}) \rightarrow \mathrm{H}^{3}(\mathcal{V}, \mathbb{K}) \rightarrow \mathrm{H}^{3}(\mathcal{V}, \mathcal{V}) \rightarrow \mathrm{H}^{3}(\mathcal{V}, \mathcal{W}) \rightarrow \ldots
$$

Concerning the third cohomology group, we have $\mathrm{H}^{3}(\mathcal{W}, \mathcal{W})=\{0\}$ as proved in the previous section. This result, together with Theorem 5.2 leads to $\mathrm{H}^{3}(\mathcal{V}, \mathcal{W})=\{0\}$. Moreover, in [26], it was shown that $\mathrm{H}^{2}(\mathcal{V}, \mathcal{W}) \cong \mathrm{H}^{2}(\mathcal{W}, \mathcal{W})$ and also $\mathrm{H}^{2}(\mathcal{W}, \mathcal{W})=\{0\}$; both results together leading to $\mathrm{H}^{2}(\mathcal{V}, \mathcal{W})=0$. Consequently, the long exact sequence $(5.1)$ above reduces for the third cohomology to:

$$
0 \rightarrow \mathrm{H}^{3}(\mathcal{V}, \mathbb{K}) \rightarrow \mathrm{H}^{3}(\mathcal{V}, \mathcal{V}) \rightarrow 0 .
$$

As $\operatorname{dim}\left(\mathrm{H}^{3}(\mathcal{V}, \mathbb{K})\right)=1$ by Theorem 5.3 , the two-term exact sequence 5.2 above yields the desired result $\operatorname{dim}\left(\mathrm{H}^{3}(\mathcal{V}, \mathcal{V})\right)=1$.

5.1. Proof of $\mathrm{H}^{3}(\mathcal{V}, \mathcal{W}) \cong \mathrm{H}^{3}(\mathcal{W}, \mathcal{W})$. We will prove this via the Hochschild-Serre spectral sequence.

Take $\mathfrak{g}=\mathcal{V}$ and $\mathfrak{h}=\mathbb{K}$ in Theorem 3.1, then $\mathfrak{g} / \mathfrak{h}=\mathcal{W}$. Hence, the second stage spectral sequence $E_{2}^{p q}$ becomes in our case:

$$
E_{2}^{p, q}=\mathrm{H}^{p}\left(\mathcal{W}, \mathrm{H}^{q}(\mathbb{K}, M)\right) \Rightarrow \mathrm{H}^{p+q}(\mathcal{V}, M) .
$$


Due to the alternating property of the cochains, we have $\mathrm{H}^{k}(\mathbb{K}, M)=0$ for $k>1$. This is true for any module $M$.

Consequently, the Hochschild-Serre spectral sequence has only two lines in our case:

$$
\begin{array}{cccc}
0 & 0 & 0 & \cdots \\
\mathrm{H}^{0}\left(\mathcal{W}, \mathrm{H}^{1}(\mathbb{K}, M)\right) & \mathrm{H}^{1}\left(\mathcal{W}, \mathrm{H}^{1}(\mathbb{K}, M)\right) & \mathrm{H}^{2}\left(\mathcal{W}, \mathrm{H}^{1}(\mathbb{K}, M)\right) & \cdots \\
\cline { 1 - 3 } \mathrm{H}^{0}\left(\mathcal{W}, \mathrm{H}^{0}(\mathbb{K}, M)\right) & \mathrm{H}^{1}\left(\mathcal{W}, \mathrm{H}^{0}(\mathbb{K}, M)\right) & \mathrm{H}^{2}\left(\mathcal{W}, \mathrm{H}^{0}(\mathbb{K}, M)\right) & \cdots
\end{array}
$$

For reference, we put the $(0,0)$-entry into a box. The entries with $p<0$ or $q<0$ are zero. In addition, the entries for $q \geq 2$ are zero because of $\mathrm{H}^{k}(\mathbb{K}, M)=0$ for $k>1$.

The second stage spectral sequence comes with differentials:

$$
d_{2}^{p, q}: E_{2}^{p, q} \longrightarrow E_{2}^{p+2, q-1}
$$

With these maps, we can take the cohomology $E_{3}^{p, q}$ of $E_{2}^{p, q}$, which gives the third page spectral sequence:

$$
E_{3}^{p, q}=\frac{\operatorname{ker} d_{2}^{p, q}}{\operatorname{im~} d_{2}^{p-2, q+1}}
$$

The third page spectral sequence has the same shape as the second page spectral sequence, meaning it too has only two lines different from zero. Again, the third page spectral sequence comes with maps:

$$
d_{3}^{p, q}: E_{3}^{p, q} \longrightarrow E_{3}^{p+3, q-2} .
$$

However, the operator $d_{3}^{p, q}$ corresponds to going three entries to the right and two entries to the bottom. Since we only have two lines different from zero, we always obtain im $d_{3}^{p, q}=0$. Moreover, the kernel of $d_{3}^{p, q}$ then corresponds to $E_{3}^{p, q}$. Therefore, we obtain $E_{3}^{p, q}=E_{4}^{p, q}=$ $\cdots=E_{\infty}^{p, q}$. We finally gathered all the necessary ingredients to prove the following result:

Theorem 5.2. The following holds true:

$$
\mathrm{H}^{3}(\mathcal{V}, \mathcal{W}) \cong \mathrm{H}^{3}(\mathcal{W}, \mathcal{W}) .
$$

Proof. If $M$ is a $\mathfrak{g}$-module, then $M$ is also a $\mathfrak{h}$-module since $\mathfrak{h} \unlhd \mathfrak{g}$ in Theorem 3.1. Then $\mathfrak{g} / \mathfrak{h}$ acts on $\mathrm{H}^{q}(\mathfrak{h}, M)$. We consider the module $M=\mathcal{W}$ in $(5.3)$, which is a $\mathcal{V}$-module and a trivial $\mathbb{K}$-module. The latter implies $\mathrm{H}^{0}(\mathbb{K}, \mathcal{W})={ }^{\mathbb{K}} \mathcal{W}=\mathcal{W}$, where ${ }^{\mathbb{K}} \mathcal{W}$ denotes the space of $\mathbb{K}$-invariants of $\mathcal{W}$.

Moreover, we have $\mathrm{H}^{1}(\mathbb{K}, \mathcal{W})=\mathcal{W}$ as every linear map $\phi \in C^{1}(\mathbb{K}, \mathcal{W})$ is a cocycle and hence $\mathrm{H}^{1}(\mathbb{K}, \mathcal{W})$ corresponds one-to-one to all linear maps $1 \mapsto \omega \in \mathcal{W}$, i.e. to all elements of $\mathcal{W}$. The $(0,0)$ and $(0,1)$ entries become: $\mathrm{H}^{0}(\mathcal{W}, \mathcal{W})=\mathcal{W}_{\mathcal{W}}=0$

Our second stage spectral sequence thus becomes:

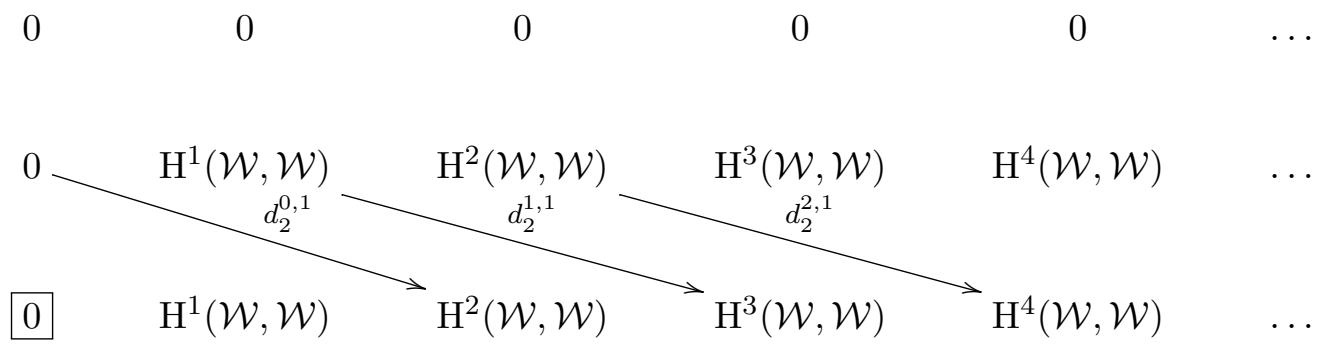


Once again, we put the $(0,0)$-entry into a box to increase readability. In order to simplify the notation, we define:

$$
\varphi_{p}:=d_{2}^{p, 1}: E_{2}^{p, 1} \longrightarrow E_{2}^{p+2,0} .
$$

Next, we take the cohomology of the sequence with respect to $\varphi_{p}$, which gives us the third page spectral sequence $E_{3}^{p, q}=E_{\infty}^{p, q}$. We will abbreviate $\mathrm{H}^{i}(\mathcal{W}, \mathcal{W})$ by $\mathrm{H}^{i}$ :

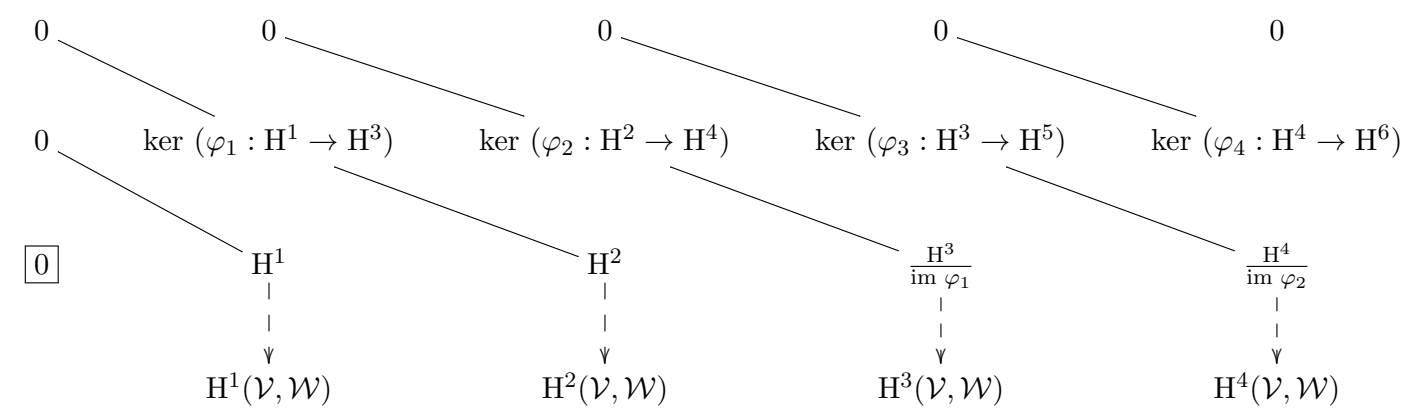

The elements $E_{2}^{p, q}$ converge to $\mathrm{H}^{p+q}(\mathfrak{g}, M)$, i.e. $\mathrm{H}^{p+q}(\mathcal{V}, \mathcal{W})$ in our case. This means that in the case under consideration, we can write the elements $\mathrm{H}^{p+q}(\mathcal{V}, \mathcal{W})$ of degree $n=p+q$ as a direct sum of the elements $E_{3}^{p, q}$ of degree $n$, i.e. the elements $E_{3}^{p, q}$ with $p+q=n$ lying on the $n$-th diagonal:

$$
\mathrm{H}^{k}(\mathcal{V}, \mathcal{W}) \cong E_{3}^{k, 0} \oplus E_{3}^{k-1,1}
$$

We see from the diagram above that for general $k \in \mathbb{N}$, we have:

$$
\mathrm{H}^{k}(\mathcal{V}, \mathcal{W}) \cong \frac{\mathrm{H}^{k}(\mathcal{W}, \mathcal{W})}{\operatorname{im} \varphi_{k-2}} \oplus \operatorname{ker}\left(\varphi_{k-1}: \mathrm{H}^{k-1}(\mathcal{W}, \mathcal{W}) \rightarrow \mathrm{H}^{k+1}(\mathcal{W}, \mathcal{W})\right)
$$

In particular, for the first three cohomology spaces we obtain,

- $\mathrm{H}^{1}(\mathcal{V}, \mathcal{W}) \cong \mathrm{H}^{1}(\mathcal{W}, \mathcal{W})$.

- $\mathrm{H}^{2}(\mathcal{V}, \mathcal{W}) \cong \mathrm{H}^{2}(\mathcal{W}, \mathcal{W}) \oplus \operatorname{ker}\left(\varphi_{1}: \mathrm{H}^{1}(\mathcal{W}, \mathcal{W}) \rightarrow \mathrm{H}^{3}(\mathcal{W}, \mathcal{W})\right)$

- $\mathrm{H}^{3}(\mathcal{V}, \mathcal{W}) \cong \frac{\mathrm{H}^{3}(\mathcal{W}, \mathcal{W})}{\operatorname{im} \varphi_{1}} \oplus \operatorname{ker}\left(\varphi_{2}: \mathrm{H}^{2}(\mathcal{W}, \mathcal{W}) \rightarrow \mathrm{H}^{4}(\mathcal{W}, \mathcal{W})\right)$

Concerning the third result, we have $\operatorname{ker}\left(\varphi_{2}: \mathrm{H}^{2}(\mathcal{W}, \mathcal{W}) \rightarrow \mathrm{H}^{4}(\mathcal{W}, \mathcal{W})\right)=0$ because $\mathrm{H}^{2}(\mathcal{W}, \mathcal{W})=\{0\}$, see [26]. Moreover, we have $\operatorname{im}\left(\varphi_{1}: \mathrm{H}^{1}(\mathcal{W}, \mathcal{W}) \rightarrow \mathrm{H}^{3}(\mathcal{W}, \mathcal{W})\right)=0$ because $\mathrm{H}^{1}(\mathcal{W}, \mathcal{W})=\{0\}$, see the appendix. Hence, we obtain the announced result.

The same techniques as the ones used in the proof above also lead to the following result:

Proposition 5.1. Let $M$ be a $\mathcal{W}$-module. Then $\forall k \in \mathbb{N}$ the following holds:

$$
\begin{aligned}
\text { If } & \mathrm{H}^{j}(\mathcal{W}, M)=0 \quad \text { for } j=k-1 \text { and } j=k-2, \\
\text { then } & \mathrm{H}^{k}(\mathcal{V}, M) \cong \mathrm{H}^{k}(\mathcal{W}, M) .
\end{aligned}
$$

Proof. The Equation (5.5) is true also in the context of general modules $M$.

Remark 5.1. Since we have $\mathrm{H}^{0}(\mathcal{W}, \mathcal{W})=0$ and also $\mathrm{H}^{1}(\mathcal{W}, \mathcal{W})=0$, we obtain from Proposition 5.1 that $\mathrm{H}^{2}(\mathcal{V}, \mathcal{W})=\mathrm{H}^{2}(\mathcal{W}, \mathcal{W})$. This has also been proved by explicit computations before in [26]. 
5.2. Analysis of $\mathrm{H}^{3}(\mathcal{W}, \mathbb{K})$ and $\mathrm{H}^{3}(\mathcal{V}, \mathbb{K})$. In this section we will prove:

Theorem 5.3. The third algebraic cohomology of the Witt and the Virasoro algebra over a field $\mathbb{K}$ with char $(\mathbb{K})=0$ with values in the trivial module $\mathbb{K}$ is one-dimensional, i.e.:

$$
\operatorname{dim}\left(\mathrm{H}^{3}(\mathcal{W}, \mathbb{K})\right)=\operatorname{dim}\left(\mathrm{H}^{3}(\mathcal{V}, \mathbb{K})\right)=1 .
$$

For the convenience of the reader, we write down the condition for a 3-cochain $\psi$ to be a cocycle with values in the trivial module:

$$
\begin{aligned}
& \left(\delta_{3} \psi\right)\left(x_{1}, x_{2}, x_{3}, x_{4}\right)=\psi\left(\left[x_{1}, x_{2}\right], x_{3}, x_{4}\right)-\psi\left(\left[x_{1}, x_{3}\right], x_{2}, x_{4}\right)+\psi\left(\left[x_{1}, x_{4}\right], x_{2}, x_{3}\right) \\
& +\psi\left(\left[x_{2}, x_{3}\right], x_{1}, x_{4}\right)-\psi\left(\left[x_{2}, x_{4}\right], x_{1}, x_{3}\right)+\psi\left(\left[x_{3}, x_{4}\right], x_{1}, x_{2}\right)=0
\end{aligned}
$$

where $x_{1}, x_{2}, x_{3}, x_{4}$ are elements of $\mathcal{W}$ or $\mathcal{V}$.

The condition for a 3-cocycle $\psi$ to be a coboundary with values in the trivial module is given by:

$$
\psi\left(x_{1}, x_{2}, x_{3}\right)=\left(\delta_{2} \phi\right)\left(x_{1}, x_{2}, x_{3}\right)=\phi\left(\left[x_{1}, x_{2}\right], x_{3}\right)+\phi\left(\left[x_{2}, x_{3}\right], x_{1}\right)+\phi\left(\left[x_{3}, x_{1}\right], x_{2}\right),
$$

where $\phi$ is a 2-cochain with values in $\mathbb{K}$ and $x_{1}, x_{2}, x_{3}$ are elements of $\mathcal{W}$ or $\mathcal{V}$.

Next, consider the following trilinear map:

$$
\Psi: \mathcal{W} \times \mathcal{W} \times \mathcal{W} \rightarrow \mathbb{K}
$$

defined on the basis elements via:

$$
\Psi\left(e_{i}, e_{j}, e_{k}\right)=(i-j)(j-k)(i-k) \delta_{i+j+k, 0},
$$

which we extend trivially to:

$$
\hat{\Psi}: \mathcal{V} \times \mathcal{V} \times \mathcal{V} \rightarrow \mathbb{K}
$$

by setting $\hat{\Psi}\left(x_{1}, x_{2}, x_{3}\right)=0$ whenever one of the elements $x_{1}, x_{2}$ or $x_{3}$ is a multiple of the central element $t$.

Proposition 5.2. The trilinear maps $\Psi$ and $\hat{\Psi}$ define non-trivial cocycle classes of $\mathrm{H}^{3}(\mathcal{W}, \mathbb{K})$ and $\mathrm{H}^{3}(\mathcal{V}, \mathbb{K})$, respectively.

Proof. By their very definition, $\Psi$ and $\hat{\Psi}$ are alternating.

A straight-forward calculation of (5.6) for a triplet of basis elements $e_{i}, e_{j}, e_{k}$ yields $\delta_{3} \Psi=0$.

Hence $\Psi$ is a three-cocycle of $\mathcal{W}$. Concerning the Virasoro algebra, we have $\delta_{3} \hat{\Psi}\left(x_{1}, x_{2}, x_{3}, x_{4}\right)=$ 0 if one of the arguments is central. If all the arguments are coming from $\mathcal{W}$, we obtain 3 $\delta_{3} \hat{\Psi}\left(x_{1}, x_{2}, x_{3}, x_{4}\right)=\delta_{3} \Psi\left(x_{1}, x_{2}, x_{3}, x_{4}\right)=0$. Thus, $\hat{\Psi}$ is a 3 -cocycle for $\mathcal{V}$. It remains to be shown that these cocycles are not trivial.

Let us assume that $\Psi$ and $\hat{\Psi}$ are coboundaries, which will lead us to a contradiction. So, let $\Phi: \mathcal{W} \times \mathcal{W} \rightarrow \mathbb{K}$ be a 2 -cochain with $\Psi=\delta_{2} \Phi$. On the one hand, evaluating $\Psi$ on the triple $e_{-1}, e_{1}, e_{0}$, we obtain by the very definition (5.8) of $\Psi$ :

$$
\Psi\left(e_{-1}, e_{1}, e_{0}\right)=2 \text {. }
$$

On the other hand, $\Psi$ being a coboundary we obtain using (5.7):

$$
\begin{aligned}
\Psi\left(e_{-1}, e_{1}, e_{0}\right) & =\Phi\left(\left[e_{-1}, e_{1}\right], e_{0}\right)+\Phi\left(\left[e_{1}, e_{0}\right], e_{-1}\right)+\Phi\left(\left[e_{0}, e_{-1}\right], e_{1}\right) \\
& =2 \Phi\left(e_{0}, e_{0}\right)-\Phi\left(e_{1}, e_{-1}\right)-\Phi\left(e_{-1}, e_{1}\right)=0 .
\end{aligned}
$$

\footnotetext{
${ }^{3}$ In abuse of notation, we use the same symbol $x$ to refer both to $x \in \mathcal{V}$ and its projection $\pi(x) \in \mathcal{W}$.
} 
Therefore, $\Psi$ cannot be a coboundary. Similarly, assume $\hat{\Psi}=\delta_{2} \hat{\Phi}$, with $\hat{\Phi}: \mathcal{V} \times \mathcal{V} \rightarrow \mathbb{K}$. Again, we obtain $\hat{\Psi}\left(e_{-1}, e_{1}, e_{0}\right)=\Psi\left(e_{-1}, e_{1}, e_{0}\right)=2$ as well as $\delta_{2} \hat{\Phi}\left(e_{-1}, e_{1}, e_{0}\right)=0$, hence $\hat{\Psi}$ cannot be a coboundary. Note that for pairs of elements of $e_{-1}, e_{1}, e_{0}$, the defining cocycle (2.3) for the central extension of $\mathcal{W}$ vanishes, hence exactly the same expression 5.10 will also appear for $\hat{\Psi}$, without any extra central terms.

We call the cocycle $\Psi$ the algebraic Godbillon-Vey cocycle. An immediate consequence of the Proposition 5.2 is:

Proposition 5.3. The third algebraic cohomology of the Witt and the Virasoro algebra over a field $\mathbb{K}$ with char $(\mathbb{K})=0$ with values in the trivial module is at least one-dimensional,

$$
\operatorname{dim}\left(\mathrm{H}^{3}(\mathcal{W}, \mathbb{K})\right) \geq 1 \text { and } \operatorname{dim}\left(\mathrm{H}^{3}(\mathcal{V}, \mathbb{K})\right) \geq 1 .
$$

Remark 5.2. In the continuous cohomology of $\mathrm{H}_{c}^{3}\left(\operatorname{Vect}\left(S^{1}\right), \mathbb{R}\right)$, there is an analytic GodbillonVey cocycle showing up. For the interested reader, we exhibit the relation to the algebraic Godbillon-Vey cocycle in the following.

Let $t$ be the coordinate along $S^{1}$. The elements of $V e c t\left(S^{1}\right)$ can be represented by functions on $S^{1}$. Assigning to the vector field $f(t) \frac{d}{d t}$ the function $f(t)$, it was shown in [12] that the continuous cohomology $\mathrm{H}_{c}^{*}\left(\operatorname{Vect}\left(S^{1}\right), \mathbb{R}\right)$ of $V e c t\left(S^{1}\right)$ with values in $\mathbb{R}$ is the free gradedcommutative algebra generated by an element $\omega$ of degree two and an element $\mathscr{G} \mathscr{V}$ of degree three. The generator of degree two is given by:

$$
\omega:\left(f \frac{d}{d t}, g \frac{d}{d t}\right) \mapsto \int_{S^{1}} \operatorname{det}\left(\begin{array}{cc}
f^{\prime} & g^{\prime} \\
f^{\prime \prime} & g^{\prime \prime}
\end{array}\right) d t
$$

while the generator of degree three is given by:

$$
\mathscr{G} \mathscr{V}:\left(f \frac{d}{d t}, g \frac{d}{d t}, h \frac{d}{d t}\right) \mapsto \int_{S^{1}} \operatorname{det}\left(\begin{array}{ccc}
f & g & h \\
f^{\prime} & g^{\prime} & h^{\prime} \\
f^{\prime \prime} & g^{\prime \prime} & h^{\prime \prime}
\end{array}\right) d t,
$$

with $f, g, h \in C^{\infty}\left(S^{1}\right)$ and the prime denoting the derivative with respect to $t$. The generator $\mathscr{G} \mathscr{V}$ in (5.12) is commonly called the Godbillon-Vey cocycle.

If one considers the complexified vector field $\tilde{e}_{n}=i e^{i n t} \frac{d}{d t}$ then

$$
\begin{aligned}
\mathscr{G V}\left(\tilde{e}_{n}, \tilde{e}_{m}, \tilde{e}_{k}\right) & =-\int_{S^{1}} \operatorname{det}\left(\begin{array}{ccc}
1 & 1 & 1 \\
n & m & k \\
n^{2} & m^{2} & k^{2}
\end{array}\right) e^{i(n+m+k) t} d t \\
& =(n-m)(n-k)(m-k) \int_{S^{1}} e^{i(n+m+k) t} d t
\end{aligned}
$$

The integral evaluates to zero if $n+m+k \neq 0$, otherwise it yields the value 1 . The expression 5.13 makes perfect sense for our algebraic generators $e_{n}$ of $\mathcal{W}$ for every field $\mathbb{K}$ with char $(\mathbb{K})=0$, and we obtain the expression 5.8 .

Before continuing, we decompose our cohomology spaces into their degree subspaces,

$$
\begin{aligned}
& \mathrm{H}^{3}(\mathcal{W}, \mathbb{K})=\bigoplus_{d \in \mathbb{Z}} \mathrm{H}_{(d)}^{3}(\mathcal{W}, \mathbb{K}), \\
& \mathrm{H}^{3}(\mathcal{V}, \mathbb{K})=\bigoplus_{d \in \mathbb{Z}} \mathrm{H}_{(d)}^{3}(\mathcal{V}, \mathbb{K})
\end{aligned}
$$


Since our module $\mathbb{K}$ is internally graded, we can use the result of Fuks 3.2 that the degree non-zero cohomology is cohomologically trivial. Consequently,

$$
\begin{aligned}
& \mathrm{H}^{3}(\mathcal{W}, \mathbb{K})=\mathrm{H}_{(0)}^{3}(\mathcal{W}, \mathbb{K}), \\
& \mathrm{H}^{3}(\mathcal{V}, \mathbb{K})=\mathrm{H}_{(0)}^{3}(\mathcal{V}, \mathbb{K}) .
\end{aligned}
$$

Phrased differently, every cocycle is cohomologous to a degree zero cocycle. Our algebraic Godbillon-Vey cocycle is clearly a non-trivial element in the $\mathrm{H}_{(0)}^{3}$-spaces.

Our proof of Theorem 5.3 shall proceed by showing that the Godbillon-Vey cocycle $\Psi$ is a generator of the spaces $\mathrm{H}^{3}(\mathcal{W}, \mathbb{K})$ and $\mathrm{H}^{3}(\mathcal{V}, \mathbb{K})$. Let $\psi$ be an arbitrary degree zero 3 -cocycle for $\mathcal{W}$ or $\mathcal{V}$. Then we set:

$$
\psi^{\prime}=\psi-\frac{\psi\left(e_{-1}, e_{1}, e_{0}\right)}{2} \Psi .
$$

By $(5.9)$, we have $\psi^{\prime}\left(e_{-1}, e_{1}, e_{0}\right)=0$. The last result to show is:

Proposition 5.4. Let $\psi$ be a 3-cocycle for $\mathcal{W}$ or $\mathcal{V}$ with $\psi\left(e_{-1}, e_{1}, e_{0}\right)=0$.

Then $\psi$ is a coboundary.

Using (5.14) and Proposition 5.4. we obtain that every cohomology class is a multiple of the algebraic Godbillon-Vey cocycle class. This implies Theorem 5.3 .

It remains to show Proposition 5.4. The proof of Proposition 5.4 is obtained by elementary algebraic manipulations. In this article, we will present only the general set-up without giving details. Details can be found ${ }^{4}$ in [4].

Let $\psi$ be a 3 -cochain and $\phi$ a 2-cochain for $\mathcal{W}$ or $\mathcal{V}$. These cochains will be given by their system of coefficients $\phi_{i, j}, b_{i}, \psi_{i, j, k}, c_{i, j} \in \mathbb{K}$ defined as follows:

$$
\begin{aligned}
& \psi\left(e_{i}, e_{j}, e_{k}\right):=\psi_{i, j, k} \quad \text { and } \quad \psi\left(e_{i}, e_{j}, t\right):=c_{i, j}, \\
& \phi\left(e_{i}, e_{j}\right):=\phi_{i, j} \quad \text { and } \quad \phi\left(e_{i}, t\right):=b_{i},
\end{aligned}
$$

with the obvious identification coming from the alternating property of the cochains.

For the convenience of the notation, we introduce the shortcut

$$
\alpha_{i}:=-\frac{1}{12}\left(i^{3}-i\right)
$$

for the cocycle giving the definition of the Virasoro algebra. Note that $\alpha_{-i}=-\alpha_{i}, \alpha_{0}=$ $\alpha_{1}=\alpha_{-1}=0$ and $\alpha_{2}=-1 / 2$.

The cocycle condition $\delta_{3} \psi=0$ and the coboundary condition $\psi=\delta_{2} \phi$ can be reformulated in terms of the coefficients, using (5.6) and (5.7) respectively. The cochain $\psi$ is a 3 -cocycle if and only if:

$$
\begin{aligned}
& 0=\delta_{3} \psi\left(e_{i}, e_{j}, e_{k}, e_{l}\right) \\
\Leftrightarrow & 0=(j-i) \psi_{i+j, k, l}-(k-i) \psi_{i+k, j, l}+(l-i) \psi_{i+l, j, k}+(k-j) \psi_{j+k, i, l} \\
& -(l-j) \psi_{l+j, i, k}+(l-k) \psi_{l+k, i, j}+\alpha_{i} \delta_{i,-j} c_{k, l}-\alpha_{i} \delta_{i,-k} c_{j, l} \\
& +\alpha_{i} \delta_{i,-l} c_{j, k}+\alpha_{j} \delta_{j,-k} c_{i, l}-\alpha_{j} \delta_{j,-l} c_{i, k}+\alpha_{k} \delta_{k,-l} c_{i, j}
\end{aligned}
$$

and

$$
0=\delta_{3} \psi\left(e_{i}, e_{j}, e_{k}, t\right) \Leftrightarrow 0=(j-i) c_{i+j, k}-(k-i) c_{i+k, j}+(k-j) c_{j+k, i} .
$$

\footnotetext{
4 or in 6
} 
The cochain $\psi$ is a coboundary if and only if:

$$
\begin{aligned}
\psi_{i, j, k}=\delta_{2} \phi\left(e_{i}, e_{j}, e_{k}\right) & =(j-i) \phi_{i+j, k}-(k-i) \phi_{i+k, j}+(k-j) \phi_{j+k, i} \\
& -\alpha_{i} \delta_{i,-j} b_{k}+\alpha_{i} \delta_{i,-k} b_{j}-\alpha_{j} \delta_{j,-k} b_{i}
\end{aligned}
$$

and

$$
c_{i, j}=\delta_{2} \phi\left(e_{i}, e_{j}, t\right)=(j-i) b_{i+j} .
$$

As we only need to consider cochains of degree zero, and since our trivial module $\mathbb{K}$ has only degree zero elements, non-zero coefficients are only possible if the indices of the said coefficients add up to zero, hence:

$$
\begin{array}{cl}
\psi_{i, j, k}=0 & i+j+k \neq 0, \\
c_{i, j}=0 & i+j \neq 0, \\
\phi_{i, j}=0 & i+j \neq 0, \\
b_{i}=0 & i \neq 0 .
\end{array}
$$

These considerations constitute the necessary ingredients to proceed with the proof of Proposition 5.4 The proof proceeds in several steps, not unlike the proof given for Theorem 4.1. In the first steps, one index of the coefficients is fixed, and results are derived for this particular level. For the coefficients $\psi_{i, j, k}$ for example, the analysis first focuses on $\psi_{i,-i, 0}$, $\psi_{i,-i-1,1}, \psi_{i,-i+1,-1}, \psi_{i,-i-2,2}$, and $\psi_{i,-i+2,-2}, \forall i \in \mathbb{Z}$, or coefficients with some permutation of these indices, which will constitute the starting points of recurrence relations between the coefficients. In the last step, adequate recurrence relations are constructed in order to express all coefficients $\psi_{i,-i-k, k} \forall i, k \in \mathbb{Z}$ in terms of a finite number of starting coefficients. The proof consists of two lemmas, which we will provide in the following without a proof. Details can be found in [4. The first lemma mostly involves a cohomological change, although in the case of the Virasoro algebra, already the cocycle condition (5.17) is used in order to put constraints on the coefficients $c_{i, j}$. The second lemma involves the cocycle condition (5.16).

Lemma 5.1. Every 3-cocycle $\psi^{\prime} \in \mathrm{H}^{3}(\mathcal{V}, \mathbb{K})$ satisfying $\psi^{\prime}\left(e_{1}, e_{-1}, e_{0}\right)=0$ is cohomologous to a 3-cocycle $\psi \in \mathrm{H}^{3}(\mathcal{V}, \mathbb{K})$ with coefficients $c_{i, j}, \psi_{i, j, k} \in \mathbb{K}$ fulfilling:

$$
c_{i, j}=\delta_{i,-j}\left(\frac{1}{6} i(i-1)(i+1) c_{2,-2}\right) \quad \text { and } \quad \psi_{i, j, 1}=0 \quad \forall i, j \in \mathbb{Z} .
$$

Every 3-cocycle $\psi^{\prime} \in \mathrm{H}^{3}(\mathcal{W}, \mathbb{K})$ satisfying $\psi^{\prime}\left(e_{1}, e_{-1}, e_{0}\right)=0$ is cohomologous to a 3-cocycle $\psi \in \mathrm{H}^{3}(\mathcal{W}, \mathbb{K})$ with coefficients $\psi_{i, j, k} \in \mathbb{K}$ fulfilling:

$$
\psi_{i, j, 1}=0 \quad \forall i, j \in \mathbb{Z} .
$$

Remark 5.3. The expression for the coefficients $c_{i, j}$ in (5.20) corresponds to the expression of the Virasoro 2-cocycle, generator of the one-dimensional $\mathrm{H}^{2}(\mathcal{W}, \mathbb{K})$ space; compare to (2.3). The fact that the two expressions agree is not surprising, because the conditions (5.19) and (5.17) involving the generator $t$ reduce to the coboundary and cocycle conditions of $\mathrm{H}^{2}(\mathcal{W}, \mathbb{K})$.

Lemma 5.2. The following holds: 
(i) Let $\psi \in \mathrm{H}^{3}(\mathcal{V}, \mathbb{K})$ be a 3 -cocycle such that:

$$
\begin{array}{rlrl}
c_{i, j} & =\delta_{i,-j} & \left(\frac{1}{6}(i-1)(i)(i+1) c_{2,-2}\right) \quad \text { and } \quad \psi_{i, j, 1}=0 \quad \forall i, j \in \mathbb{Z} . \\
\text { Then } \quad c_{i, j} & =0 \quad \forall i, j \in \mathbb{Z} \quad \text { and } \quad \psi_{i, j, k}=0 \quad \forall i, j, k \in \mathbb{Z} .
\end{array}
$$

(ii) Let $\psi \in \mathrm{H}^{3}(\mathcal{W}, \mathbb{K})$ be a 3 -cocycle such that:

$$
\begin{aligned}
& \psi_{i, j, 1}=0 & \forall i, j \in \mathbb{Z} . \\
\text { Then } & \psi_{i, j, k}=0 & \forall i, j, k \in \mathbb{Z} .
\end{aligned}
$$

Proof of Proposition 5.4: Lemma 5.1 tells us that we can always perform a cohomological change of a 3 -cocycle in $\mathrm{H}^{3}(\mathcal{W}, \mathbb{K})$ or $\mathrm{H}^{3}(\mathcal{V}, \mathbb{K})$ satisfying $\psi\left(e_{-1}, e_{1}, e_{0}\right)=0$ such that we obtain a cohomological equivalent 3 -cocycle with coefficients fulfilling the assumptions of Lemma 5.2. Lemma 5.2 tells us that all the coefficients of the 3-cocycle satisfying $\psi\left(e_{-1}, e_{1}, e_{0}\right)=0$ are zero. This allows to conclude.

Remark 5.4. In the proof of Proposition 5.4, $\mathcal{W}$ and $\mathcal{V}$ are treated in a similar manner, yielding $\mathrm{H}^{3}(\mathcal{W}, \mathbb{K}) \cong \mathrm{H}^{3}(\mathcal{V}, \mathbb{K})$. The reader might have gotten the impression that this could also be true for other cohomology spaces. However, already

$$
1=\operatorname{dim}\left(\mathrm{H}^{2}(\mathcal{W}, \mathbb{K})\right) \neq \operatorname{dim}\left(\mathrm{H}^{2}(\mathcal{V}, \mathbb{K})\right)=0,
$$

shows that the underlying structure is more delicate. The Hochschild-Serre spectral sequence with the trivial module $M=\mathbb{K}$ in (5.3) gives more information.

$$
\text { 6. Analysis of } \mathrm{H}^{1}\left(\mathcal{W}, \mathcal{F}^{\lambda}\right), \mathrm{H}^{2}\left(\mathcal{W}, \mathcal{F}^{\lambda}\right), \mathrm{H}^{1}\left(\mathcal{V}, \mathcal{F}^{\lambda}\right) \text { And } \mathrm{H}^{2}\left(\mathcal{V}, \mathcal{F}^{\lambda}\right)
$$

In the previous sections, we focused on the low-dimensional algebraic cohomology with values in the adjoint and the trivial modules. However, the adjoint and the trivial module are only specific modules that are included in a larger, actually infinite family of modules, called the tensor densities modules $\mathcal{F}^{\lambda}$.

The action of the Witt algebra on the general tensor densities modules $\mathcal{F}^{\lambda}$ is given by:

$$
e_{n} \cdot f_{m}^{\lambda}=(m+\lambda n) f_{n+m}^{\lambda}, \quad n, m \in \mathbb{Z},
$$

where $e_{n} \in \mathcal{W}, f_{m}^{\lambda} \in \mathcal{F}^{\lambda}$ and $\lambda \in \mathbb{C}$. By defining the degree of an element as $\operatorname{deg}\left(f_{m}^{\lambda}\right):=m$, $\mathcal{F}^{\lambda}$ becomes an internally graded module over $\mathcal{W}$.

In the geometrical realization of the Witt algebra on $\mathbb{C P}^{1}$, described in Section 2 , and for integer $\lambda$, the elements $f_{m}^{\lambda}$ can be interpreted as meromorphic forms of weight $\lambda$ and written as $f_{m}^{\lambda}=z^{m-\lambda}(d z)^{\lambda}$, hence the name. Thus, $\lambda=0$ for example corresponds to meromorphic functions, which include the module $\mathbb{K}$ viewed as constant functions. Moreover, the adjoint module $\lambda=-1$ is given by meromorphic vector fields, and $\lambda=1$ corresponds to meromorphic differential 1-forms. Also for half-integer $\lambda$, a geometrical interpretation can be given. However, in our work we consider $\lambda \in \mathbb{C}$, since we work on a purely algebraic level.

This section concentrates on the results obtained for the first and second algebraic cohomology with values in general tensor densities modules. In the present article, we only provide a sketch of the proofs, as the techniques used are similar to the ones we already presented in this article. For more details on the proofs, we refer the reader to [4]. 
6.1. Results for the Witt algebra. The results mainly split into two categories: The vanishing of the low-dimensional cohomology for all $\lambda$ in $\mathbb{C}$ except for a few exceptional values of $\lambda$, and the non-zero dimension of the cohomology for these exceptional values of $\lambda$. In case of the non-vanishing cohomology, we provide explicit expressions for the generating cocycles.

Due to the result (3.2) of Fuks, it is sufficient to consider the degree zero part of the cohomology of the Witt algebra. We can write a degree zero 2-cochain and a degree zero 1-cochain with values in $\mathcal{F}^{\lambda}$ as follows: $\psi^{\lambda}\left(e_{i}, e_{j}\right)=\psi_{i, j}^{\lambda} f_{i+j}^{\lambda}$ and $\phi^{\lambda}\left(e_{i}\right)=\phi_{i}^{\lambda} f_{i}^{\lambda}$, respectively, with adequate coefficients $\psi_{i, j}^{\lambda}, \phi_{i}^{\lambda} \in \mathbb{K}$ and $\psi_{i, j}^{\lambda}=-\psi_{j, i}^{\lambda}$, the $e_{i} \in \mathcal{W}$ and the $f_{i}^{\lambda} \in \mathcal{F}^{\lambda}$. Writing down the appropriate coboundary and cocycle conditions in terms of these coefficients, one uses these to construct recurrence relations in order to express all the coefficients in terms of a finite number of starting coefficients. Analyzing the relations between the starting coefficients, one can either deduce the vanishing of the cohomology or derive an upper bound for its dimension. To derive a lower bound for the dimension, one can inspire from results of the continuous cohomology, especially for the second cohomology, the generating cocycles of which are known, see the article by Ovsienko and Roger [23] for the situation on $S^{1}$ and the article by Wagemann [29] for the situation on $\mathbb{C P}^{1}$.

Theorem 6.1. The first algebraic cohomology of the Witt algebra $\mathcal{W}$ over a field $\mathbb{K}$ with $\operatorname{char}(\mathbb{K})=0$ and values in $\mathcal{F}^{\lambda}$ with $\lambda \in \mathbb{C} \backslash\{0,1,2\}$ vanishes, i.e.

$$
\forall \lambda \in \mathbb{C} \backslash\{0,1,2\}: \mathrm{H}^{1}\left(\mathcal{W}, \mathcal{F}^{\lambda}\right)=\{0\} .
$$

Theorem 6.2. The first algebraic cohomology of the Witt algebra $\mathcal{W}$ over a field $\mathbb{K}$ with $\operatorname{char}(\mathbb{K})=0$ and values in $\mathcal{F}^{\lambda}$ with $\lambda=\{0,1,2\}$ is given by:

$$
\begin{gathered}
\operatorname{dim}\left(\mathrm{H}^{1}\left(\mathcal{W}, \mathcal{F}^{0}\right)\right)=2, \\
\operatorname{dim}\left(\mathrm{H}^{1}\left(\mathcal{W}, \mathcal{F}^{1}\right)\right)=\operatorname{dim}\left(\mathrm{H}^{1}\left(\mathcal{W}, \mathcal{F}^{2}\right)\right)=1 .
\end{gathered}
$$

Remarks about the proof. The proof follows in two step by first deriving an upper bound for the dimension, then a lower bound. Details can be found in [4]. Here, we will only give representing cocycles of the cocycle classes that generate the cohomology in question. The two generating cocycles of $\mathrm{H}^{1}\left(\mathcal{W}, \mathcal{F}^{0}\right)$ are given by:

$$
\psi^{0,(1)}\left(e_{i}\right)=\psi_{i}^{0,(1)} f_{i}^{0}:=i f_{i}^{0} \quad \text { and } \quad \psi^{0,(2)}\left(e_{i}\right)=\psi_{i}^{0,(2)} f_{i}^{0}:=(i-1) f_{i}^{0},
$$

with $e_{i} \in \mathcal{W}, \psi_{i}^{0,(1)}, \psi_{i}^{0,(2)} \in \mathbb{K}$ and $f_{i}^{0} \in \mathcal{F}^{0}$. The first upper index refers to the value of $\lambda$, the second upper index labels the generating cocycle under consideration. The generating cocycle of $\mathrm{H}^{1}\left(\mathcal{W}, \mathcal{F}^{1}\right)$ is given by:

$$
\psi^{1}\left(e_{i}\right)=\psi_{i}^{1} f_{i}^{1}:=i(i-1) f_{i}^{1},
$$

with $e_{i} \in \mathcal{W}, \psi_{i}^{1,(1)}, \psi_{i}^{1,(2)} \in \mathbb{K}$ and $f_{i}^{1} \in \mathcal{F}^{1}$. Finally, the generating cocycle of $\mathrm{H}^{1}\left(\mathcal{W}, \mathcal{F}^{2}\right)$ is given by:

$$
\psi^{2}\left(e_{i}\right)=\psi_{i}^{2} f_{i}^{2}:=i(i+1)(i-1) f_{i}^{2},
$$

with $e_{i} \in \mathcal{W}, \psi_{i}^{2,(1)}, \psi_{i}^{2,(2)} \in \mathbb{K}$ and $f_{i}^{2} \in \mathcal{F}^{2}$. These cocycles can be found by solving recurrence relations appearing in the proof. 
Theorem 6.3. The second algebraic cohomology of the Witt algebra $\mathcal{W}$ over a field $\mathbb{K}$ with $\operatorname{char}(\mathbb{K})=0$ and values in $\mathcal{F}^{\lambda}$ with $\lambda \in \mathbb{C} \backslash\{0,1,2,5,7\}$ vanishes, i.e.

$$
\forall \lambda \in \mathbb{C} \backslash\{0,1,2,5,7\}: \mathrm{H}^{2}\left(\mathcal{W}, \mathcal{F}^{\lambda}\right)=\{0\} .
$$

Theorem 6.4. The second algebraic cohomology of the Witt algebra $\mathcal{W}$ over a field $\mathbb{K}$ with $\operatorname{char}(\mathbb{K})=0$ and values in $\mathcal{F}^{\lambda}$ with $\lambda \in\{0,1,2,5,7\}$ is given by:

$$
\begin{gathered}
\operatorname{dim}\left(\mathrm{H}^{2}\left(\mathcal{W}, \mathcal{F}^{0}\right)\right)=\operatorname{dim}\left(\mathrm{H}^{2}\left(\mathcal{W}, \mathcal{F}^{1}\right)\right)=\operatorname{dim}\left(\mathrm{H}^{2}\left(\mathcal{W}, \mathcal{F}^{2}\right)\right)=2, \\
\operatorname{dim}\left(\mathrm{H}^{2}\left(\mathcal{W}, \mathcal{F}^{5}\right)\right)=\operatorname{dim}\left(\mathrm{H}^{2}\left(\mathcal{W}, \mathcal{F}^{7}\right)\right)=1
\end{gathered}
$$

Remarks about the proof. The generating cocycles of $\mathrm{H}^{2}\left(\mathcal{W}, \mathcal{F}^{0}\right)$ are given by:

$$
\begin{aligned}
& \psi^{0,(1)}\left(e_{i}, e_{j}\right)=\psi_{i, j}^{0,(1)} f_{i+j}:=(j-i) f_{i+j}, \\
& \psi^{0,(2)}\left(e_{i}, e_{j}\right)=\psi_{i, j}^{0,(2)} f_{i+j}:=\left(i j^{2}-i^{2} j\right) \delta_{i+j, 0} f_{i+j},
\end{aligned}
$$

with $e_{i} \in \mathcal{W}, \psi_{i, j}^{0,(1)}, \psi_{i, j}^{0,(2)} \in \mathbb{K}$ and $f_{i}^{0} \in \mathcal{F}^{0}$. For $\mathrm{H}^{2}\left(\mathcal{W}, \mathcal{F}^{1}\right)$, generating cocycles are given by:

$$
\begin{aligned}
& \psi^{1,(1)}\left(e_{i}, e_{j}\right)=\psi_{i, j}^{1,(1)} f_{i+j}^{1}:=\left(i j^{2}-i^{2} j\right) f_{i+j}^{1}, \\
& \psi^{1,(2)}\left(e_{i}, e_{j}\right)=\psi_{i, j}^{1,(2)} f_{i+j}^{1}:=\left(j^{2}-i^{2}\right) f_{i+j}^{1},
\end{aligned}
$$

with $e_{i} \in \mathcal{W}, \psi_{i, j}^{1,(1)}, \psi_{i, j}^{1,(2)} \in \mathbb{K}$ and $f_{i}^{1} \in \mathcal{F}^{1}$. For $\mathrm{H}^{2}\left(\mathcal{W}, \mathcal{F}^{2}\right)$, generating cocycles are given by:

$$
\begin{aligned}
& \psi^{2,(1)}\left(e_{i}, e_{j}\right)=\psi_{i, j}^{2,(1)} f_{i+j}^{2}:=\left(i j^{3}-i^{3} j\right) f_{i+j}^{2}, \\
& \psi^{2,(2)}\left(e_{i}, e_{j}\right)=\psi_{i, j}^{2,(2)} f_{i+j}^{2}:=\left(j^{3}-i^{3}\right) f_{i+j}^{2},
\end{aligned}
$$

with $e_{i} \in \mathcal{W}, \psi_{i, j}^{2,(1)}, \psi_{i, j}^{2,(2)} \in \mathbb{K}$ and $f_{i}^{2} \in \mathcal{F}^{2}$. For $\mathrm{H}^{2}\left(\mathcal{W}, \mathcal{F}^{5}\right)$, a generating cocycle is given by:

$$
\psi^{5}\left(e_{i}, e_{j}\right)=\psi_{i, j}^{5} f_{i+j}^{5}:=\left(i^{3} j^{4}-i^{4} j^{3}\right) f_{i+j}^{5} .
$$

with $e_{i} \in \mathcal{W}, \psi_{i, j}^{5} \in \mathbb{K}$ and $f_{i}^{5} \in \mathcal{F}^{5}$. Finally, for $\mathrm{H}^{2}\left(\mathcal{W}, \mathcal{F}^{7}\right)$, a generating cocycle is given by:

$$
\psi^{7}\left(e_{i}, e_{j}\right)=\psi_{i, j}^{7} f_{i+j}^{7}:=2\left(i^{3} j^{6}-i^{6} j^{3}\right)-9\left(i^{4} j^{5}-i^{5} j^{4}\right) f_{i+j}^{7} .
$$

with $e_{i} \in \mathcal{W}, \psi_{i, j}^{7} \in \mathbb{K}$ and $f_{i}^{7} \in \mathcal{F}^{7}$.

Remark 6.1. The cocycle $\psi^{0,(2)}\left(e_{i}, e_{j}\right)=\left(i j^{2}-i^{2} j\right) \delta_{i+j, 0} f_{i+j}$ appearing for $\lambda=0$ is similar to the Virasoro 2-cocycle defined in 2.3. This is not surprising since $\mathbb{K} \subset \mathcal{F}^{0}$.

Remark 6.2. For $\lambda=5$ and $\lambda=7$, a more general expression can be found for the generating cocycles by solving recurrence relations appearing in the proof:

$$
\begin{aligned}
& \psi^{5}\left(e_{i}, e_{j}\right)=\psi_{i, j}^{5} f_{i+j}^{5}=(i-1) i(i+1)(i-k) k(k-1)(k+1) f_{i+j}^{5}, \\
& \psi^{7}\left(e_{i}, e_{j}\right)=\psi_{i, j}^{7} f_{i+j}^{7}=i\left(i^{2}-1\right)(i-k) k\left(k^{2}-1\right)\left(2 i^{2}-7 i k+16+2 k^{2}\right) f_{i+j}^{7} .
\end{aligned}
$$

However, only the terms with maximal degree are of interest, the terms of lower degree are coboundary terms.

Remark 6.3. For certain values of $\lambda$, results for the third algebraic cohomology of the Witt algebra with values in tensor densities modules are known, see [4 for details. 


\subsection{Results for the Virasoro algebra.}

Theorem 6.5. The first and the second algebraic cohomology of the Virasoro algebra $\mathcal{V}$ over a field $\mathbb{K}$ with char $(\mathbb{K})=0$ and values in $\mathcal{F}^{\lambda}$ is given by:

$$
\begin{gathered}
\mathrm{H}^{1}\left(\mathcal{V}, \mathcal{F}^{\lambda}\right)=\{0\} \quad \forall \lambda \in \mathbb{C} \backslash\{0,1,2\}, \\
\operatorname{dim}\left(\mathrm{H}^{1}\left(\mathcal{V}, \mathcal{F}^{0}\right)\right)=2 \quad \text { and } \quad \operatorname{dim}\left(\mathrm{H}^{1}\left(\mathcal{V}, \mathcal{F}^{1}\right)\right)=\operatorname{dim}\left(\mathrm{H}^{1}\left(\mathcal{V}, \mathcal{F}^{2}\right)\right)=1, \\
\mathrm{H}^{2}\left(\mathcal{V}, \mathcal{F}^{\lambda}\right)=\{0\} \quad \forall \lambda \in \mathbb{C} \backslash\{0,1,2,5,7\}, \\
\operatorname{dim}\left(\mathrm{H}^{2}\left(\mathcal{V}, \mathcal{F}^{1}\right)\right)=\operatorname{dim}\left(\mathrm{H}^{2}\left(\mathcal{V}, \mathcal{F}^{2}\right)\right)=2, \\
\operatorname{dim}\left(\mathrm{H}^{2}\left(\mathcal{V}, \mathcal{F}^{0}\right)\right)=\operatorname{dim}\left(\mathrm{H}^{2}\left(\mathcal{V}, \mathcal{F}^{5}\right)\right)=\operatorname{dim}\left(\mathrm{H}^{2}\left(\mathcal{V}, \mathcal{F}^{7}\right)\right)=1 .
\end{gathered}
$$

The proof follows in two steps. In a first step, we prove the statements given in Proposition 6.1 below, which are an immediate consequence of Proposition 5.1. In a second step, we comment on the proof of the statements given in Proposition 6.2 below, which is more computational. We will not provide details of it here.

\section{Proposition 6.1.}

$$
\begin{gathered}
\mathrm{H}^{1}\left(\mathcal{W}, \mathcal{F}^{\lambda}\right) \cong \mathrm{H}^{1}\left(\mathcal{V}, \mathcal{F}^{\lambda}\right) \quad \forall \lambda \in \mathbb{C} \backslash\{0\} . \\
\mathrm{H}^{2}\left(\mathcal{W}, \mathcal{F}^{\lambda}\right) \cong \mathrm{H}^{2}\left(\mathcal{V}, \mathcal{F}^{\lambda}\right) \quad \forall \lambda \in \mathbb{C} \backslash\{0,1,2\} .
\end{gathered}
$$

Proof. For $\lambda \neq 0$, we have $\mathrm{H}^{0}\left(\mathcal{W}, \mathcal{F}^{\lambda}\right)=0$. Thus, Proposition 5.1 immediately yields (6.7a). Besides, we have $\mathrm{H}^{1}\left(\mathcal{W}, \mathcal{F}^{\lambda}\right)=0$ for $\lambda \notin\{0,1,2\}$. Hence, Proposition 5.1 also immediately yields $6.7 \mathrm{~b}$.

Proposition 6.2. The dimension of the first algebraic cohomology with values in the module $\mathcal{F}^{0}$ and the dimensions of the second algebraic cohomology with values in the modules $\mathcal{F}^{0}$, $\mathcal{F}^{1}$ and $\mathcal{F}^{2}$ of the Virasoro algebra are given by:

$$
\begin{gathered}
\operatorname{dim}\left(\mathrm{H}^{1}\left(\mathcal{V}, \mathcal{F}^{0}\right)\right)=2, \\
\operatorname{dim}\left(\mathrm{H}^{2}\left(\mathcal{V}, \mathcal{F}^{0}\right)\right)=1 \text { and } \operatorname{dim}\left(\mathrm{H}^{2}\left(\mathcal{V}, \mathcal{F}^{1}\right)\right)=\operatorname{dim}\left(\mathrm{H}^{2}\left(\mathcal{V}, \mathcal{F}^{2}\right)\right)=2 .
\end{gathered}
$$

Remarks about the proof. The Formula $(5.5)$ for the module $\mathcal{F}^{0}$ gives an upper bound of three and a lower bound of two for the dimension of $\mathrm{H}^{1}\left(\mathcal{V}, \mathcal{F}^{0}\right)$. The proof of $\operatorname{dim}\left(\mathrm{H}^{1}\left(\mathcal{V}, \mathcal{F}^{0}\right)\right)=2$ consists in writing down the coboundary and the cocycle conditions for $\mathrm{H}^{1}\left(\mathcal{V}, \mathcal{F}^{0}\right)$ in terms of the coefficients of the cocycles and cochains. The aim is then to build recurrence relations and express all the coefficients in terms of a few generating coefficients. In the case of $\mathrm{H}^{1}\left(\mathcal{V}, \mathcal{F}^{0}\right)$, the number of generating coefficients can be reduced to two, which is enough to conclude since we already have a lower bound of two for the dimension due to the Hochschild-Serre spectral sequence.

In the case of $\mathrm{H}^{2}\left(\mathcal{V}, \mathcal{F}^{0}\right)$, the Hochschild-Serre spectral sequence gives an upper bound of four and a lower bound of one for the dimension of $\mathrm{H}^{2}\left(\mathcal{V}, \mathcal{F}^{0}\right)$. The procedure using recurrence relations allows to reduce the number of generating coefficients to one, which is enough to conclude.

Similarly, for $\mathrm{H}^{2}\left(\mathcal{V}, \mathcal{F}^{1}\right)$ and $\mathrm{H}^{2}\left(\mathcal{V}, \mathcal{F}^{2}\right)$, the proof of Proposition 6.1 gives a lower bound of two and an upper bound of three for the dimension. By similar computations, one can indeed show that the number of generating coefficients is two for both $\mathrm{H}^{2}\left(\mathcal{V}, \mathcal{F}^{1}\right)$ and $\mathrm{H}^{2}\left(\mathcal{V}, \mathcal{F}^{2}\right)$.

Proof of Theorem 6.5. Clearly, Proposition 6.1 and Proposition 6.2, together with Theorems 6.1, 6.2, 6.3 and 6.4 prove Theorem 6.5 . 


\section{Appendix A. The First COHomology with Values in the ADJoint module}

In this appendix, we provide an explicit proof of the vanishing of the first cohomology, $\mathrm{H}^{1}(\mathcal{W}, \mathcal{W})=\{0\}$ and $\mathrm{H}^{1}(\mathcal{V}, \mathcal{V})=\{0\}$

A.1. The Witt algebra. In this section, we analyze the first cohomology group of the Witt algebra with values in the adjoint module. It is already known that all derivations of the Witt algebra are inner derivations, see Zhu and Meng [32], i.e. $\mathrm{H}^{1}(\mathcal{W}, \mathcal{W})=0$ in the language of cohomological algebra. However, we shall prove this result again by using our algebraic techniques developed here, in order to introduce our notation and to provide a warm-up example of the used procedures. The main aim of this section is to prove the following theorem:

Theorem A.1. The first algebraic cohomology of the Witt algebra $\mathcal{W}$ over a field $\mathbb{K}$ with char $(\mathbb{K})=0$ and values in the adjoint module vanishes, i.e.

$$
\mathrm{H}^{1}(\mathcal{W}, \mathcal{W})=\{0\}
$$

The proof follows in two steps, the first step concentrating on the non-zero degree cohomology of the Witt algebra, the second step focusing on the degree zero cohomology.

Recall that the condition for a 1-cochain $\psi$ to be a 1-cocycle is given by:

$$
\delta_{1} \psi\left(x_{1}, x_{2}\right)=0=\psi\left(\left[x_{1}, x_{2}\right]\right)-\left[x_{1}, \psi\left(x_{2}\right)\right]-\left[\psi\left(x_{1}\right), x_{2}\right],
$$

with $x_{1}, x_{2} \in \mathcal{W}$. The condition for a 1 -cocycle $\psi \in \mathrm{H}^{1}(\mathcal{W}, \mathcal{W})$ to be a coboundary is given by:

$$
\psi(x)=\left(\delta_{0} \phi\right)(x)=-x \cdot \phi=[\phi, x],
$$

with $x \in \mathcal{W}$ and $\phi \in \mathrm{C}^{0}(\mathcal{W}, \mathcal{W})=\mathcal{W}$. As the values are taken in the adjoint module, we have $\cdot=[.,$.$] .$

A.1.1. The non-zero degree cohomology of the Witt algebra.

Proposition A.1. The following holds:

$$
\mathrm{H}_{(d)}^{1}(\mathcal{W}, \mathcal{W})=\{0\} \quad \text { for } d \neq 0 \quad \text { and } \quad \mathrm{H}^{1}(\mathcal{W}, \mathcal{W})=\mathrm{H}_{(0)}^{1}(\mathcal{W}, \mathcal{W})
$$

Proof. Let $\psi \in \mathrm{H}_{(d \neq 0)}^{1}(\mathcal{W}, \mathcal{W})$.

Let us perform a cohomological change $\psi^{\prime}=\psi-\delta_{0} \phi$ with the following 0-cochain $\phi$ :

$$
\phi=-\frac{1}{d} \psi\left(e_{0}\right) \in \mathcal{W} \Rightarrow\left(\delta_{0} \phi\right)(x)=\frac{1}{d}\left[x, \psi\left(e_{0}\right)\right]
$$

which gives us:

$$
\psi^{\prime}(x)=\psi(x)-\left(\delta_{0} \phi\right)(x)=\psi(x)+\frac{1}{d}\left[\psi\left(e_{0}\right), x\right]
$$

Hence,

$$
\psi^{\prime}\left(e_{0}\right)=\psi\left(e_{0}\right)+\frac{1}{d}\left[\psi\left(e_{0}\right), e_{0}\right]=\psi\left(e_{0}\right)-\frac{1}{d} \operatorname{deg}\left(\psi\left(e_{0}\right)\right) \psi\left(e_{0}\right)=\psi\left(e_{0}\right)-\frac{1}{d} d \psi\left(e_{0}\right)=0 .
$$


We thus have $\psi^{\prime}\left(e_{0}\right)=0$.

Next, let us write down the cocycle condition for $\psi^{\prime}$ on the doublet $\left(x, e_{0}\right)$ for $x \in \mathcal{W}$ :

$$
\begin{aligned}
& 0=\psi^{\prime}\left(\left[x, e_{0}\right]\right)-\underbrace{\left[x, \psi^{\prime}\left(e_{0}\right)\right]}_{=0}-\left[\psi^{\prime}(x), e_{0}\right] \Leftrightarrow 0=\psi^{\prime}(-\operatorname{deg}(x) x)+\operatorname{deg}\left(\psi^{\prime}(x)\right) \psi^{\prime}(x) \\
& \Leftrightarrow 0=-\operatorname{deg}(x) \psi^{\prime}(x)+(\operatorname{deg}(x)+d) \psi^{\prime}(x) \Leftrightarrow 0=d \psi^{\prime}(x) .
\end{aligned}
$$

As $d \neq 0$, we get $\psi^{\prime}(x)=0 \forall x \in \mathcal{W}$, meaning that $\psi$ is a coboundary on $\mathcal{W}$. We conclude that the first cohomology of the Witt algebra reduces to the degree zero cohomology, in accordance with the result $(3.2)$.

\section{A.1.2. The degree zero cohomology for the Witt algebra.}

Proposition A.2. The following holds:

$$
\mathrm{H}_{(0)}^{1}(\mathcal{W}, \mathcal{W})=\{0\}
$$

Proof. Let $\psi$ be a degree zero 1-cocycle, i.e. we can write it as $\psi\left(e_{i}\right)=\psi_{i} e_{i}$ with suitable coefficients $\psi_{i} \in \mathbb{K}$. Consider the following 0 -cochain $\phi=\psi_{1} e_{0}$. The coboundary condition for $\phi$ gives:

$$
\left(\delta_{0} \phi\right)\left(e_{i}\right)=\left[\phi, e_{i}\right]=i \psi_{1} e_{i} .
$$

The cohomological change $\psi^{\prime}=\psi-\delta_{0} \phi$ leads to $\psi_{1}^{\prime}=0$. In the following, we will work with a 1-cocycle normalized to $\psi_{1}^{\prime}=0$, although we will drop the apostrophe in order to augment readability.

The 1-cocycle condition for $\psi$ on the doublet $\left(e_{i}, e_{j}\right)$ becomes:

$$
\begin{aligned}
0 & =\psi\left(\left[e_{i}, e_{j}\right]\right)-\left[e_{i}, \psi\left(e_{j}\right)\right]-\left[\psi\left(e_{i}\right), e_{j}\right] \\
\Leftrightarrow 0 & =(j-i)\left(\psi_{i+j}-\psi_{j}-\psi_{i}\right) .
\end{aligned}
$$

For $j=1$ and $i=0$, we obtain from the 1-cocycle condition: $\psi_{0}=0$.

For $j=1$ and $i<0$ decreasing, we obtain from the 1-cocycle condition: $\psi_{i}=\psi_{i+1}=0$.

For $j=1$ and $i>1$ increasing, we obtain from the 1-cocycle condition: $\psi_{i+1}=\psi_{i}=\psi_{2}$, where the value of $\psi_{2}$ is unknown for the moment.

Next, taking $j=2$ and for example $i=3$, we obtain:

$$
\begin{aligned}
& \psi_{5}-\psi_{2}-\psi_{3}=0 \\
\Leftrightarrow & \psi_{2}-\psi_{2}-\psi_{2}=0 \text { as we have } \psi_{i}=\psi_{2} \forall i>1 \\
\Leftrightarrow & \psi_{2}=0 .
\end{aligned}
$$

All in all, we conclude $\psi_{i}=0 \forall i \in \mathbb{Z}$.

This concludes the proof of Theorem A.1.

A.2. The Virasoro algebra. We already showed $\mathrm{H}^{1}(\mathcal{W}, \mathcal{W})=\{0\}$, and we also have $\mathrm{H}^{1}(\mathcal{V}, \mathbb{K})=\{0\}$, see Section 3.3 . These results will be used in the proof of $\mathrm{H}^{1}(\mathcal{V}, \mathcal{V})=\{0\}$ based on long exact sequences. This time we will focus only on the degree zero cohomology accordingly to the result $(3.2)$. 
Theorem A.2. The first algebraic cohomology of the Virasoro algebra $\mathcal{V}$ over a field $\mathbb{K}$ with $\operatorname{char}(\mathbb{K})=0$ and values in the adjoint module vanishes, i.e.

$$
\mathrm{H}^{1}(\mathcal{V}, \mathcal{V})=\{0\}
$$

Proof. For the first cohomology, the relevant part of the long exact sequence (5.1) looks as follows:

$$
\cdots \longrightarrow \mathrm{H}^{1}(\mathcal{V}, \mathbb{K}) \longrightarrow \mathrm{H}^{1}(\mathcal{V}, \mathcal{V}) \stackrel{\nu_{*}}{\longrightarrow} \mathrm{H}^{1}(\mathcal{V}, \mathcal{W}) \longrightarrow \ldots
$$

where $\nu: \mathcal{V} \rightarrow \mathcal{W}$ is the projection. Since we already have $\mathrm{H}^{1}(\mathcal{V}, \mathbb{K})=\{0\}$ and also $\mathrm{H}^{1}(\mathcal{W}, \mathcal{W})=\{0\}$, it suffices to prove $\mathrm{H}^{1}(\mathcal{V}, \mathcal{W}) \cong \mathrm{H}^{1}(\mathcal{W}, \mathcal{W})$ in order to conclude. This follows immediately from the Hochschild-Serre spectral sequence as shown in the proof of Theorem 5.2. Nevertheless, since our intention is to to stay elementary in this appendix, we prefer to present additionally a more direct proof in the following.

The proof consists of two steps. First, we will compare the cocycles of $Z^{1}(\mathcal{V}, \mathcal{W})$ to the cocycles of $Z^{1}(\mathcal{W}, \mathcal{W})$. In the second step, we will compare the coboundaries of $B^{1}(\mathcal{V}, \mathcal{W})$ to the ones of $B^{1}(\mathcal{W}, \mathcal{W})$.

Let $\hat{\psi}: \mathcal{V} \rightarrow \mathcal{W}$ be a cocycle of $Z^{1}(\mathcal{V}, \mathcal{W})$. Our aim is to show that the restriction of this cocycle to $\mathcal{W}$, i.e. $\psi:=\left.\hat{\psi}\right|_{\mathcal{W}}: \mathcal{W} \rightarrow \mathcal{W}$, is a cocycle of $Z^{1}(\mathcal{W}, \mathcal{W})$. Let $x_{1}, x_{2} \in \mathcal{V}$. Writing the Virasoro product $[\cdot, \cdot]_{\mathcal{V}}$ in terms of the Witt product $[\cdot, \cdot]_{\mathcal{W}}$ and the 2-cocycle $\alpha(\cdot, \cdot)$ giving the central extension, i.e. $[\cdot, \cdot]_{\mathcal{V}}=[\cdot, \cdot]_{\mathcal{W}}+\alpha(\cdot, \cdot) \cdot t$, the cocycle condition for $\hat{\psi}$ becomes:

$$
\begin{aligned}
& 0=\left(\delta_{1}^{\mathcal{V}} \hat{\psi}\right)\left(x_{1}, x_{2}\right)=\hat{\psi}\left(\left[x_{1}, x_{2}\right]^{\mathcal{V}}\right)-x_{1} \cdot \hat{\psi}\left(x_{2}\right)+x_{2} \cdot \hat{\psi}\left(x_{1}\right) \\
& \Leftrightarrow 0=\left(\delta_{1}^{\mathcal{V}} \hat{\psi}\right)\left(x_{1}, x_{2}\right)=\hat{\psi}\left(\left[x_{1}, x_{2}\right]^{\mathcal{W}}\right)+\alpha\left(x_{1}, x_{2}\right) \hat{\psi}(t)-\left[x_{1}, \hat{\psi}\left(x_{2}\right)\right]^{\mathcal{W}}+\left[x_{2}, \hat{\psi}\left(x_{1}\right)\right]^{\mathcal{W}} \\
& \Leftrightarrow 0=\left(\delta_{1}^{\mathcal{V}} \hat{\psi}\right)\left(x_{1}, x_{2}\right)=\left(\delta_{1}^{\mathcal{W}} \hat{\psi}\right)\left(x_{1}, x_{2}\right)+\alpha\left(x_{1}, x_{2}\right) \hat{\psi}(t) .
\end{aligned}
$$

Since we are considering degree-zero cocycles, the cocycle $\hat{\psi}$ evaluated on the central element reads as follows:

$$
\hat{\psi}(t)=c e_{0},
$$

for suitable $c \in \mathbb{K}$. Next, let us insert this expression into the cocycle condition for $\left(e_{1}, t\right)$, which yields:

$$
\begin{aligned}
& \left(\delta_{1}^{\mathcal{V}} \hat{\psi}\right)\left(e_{1}, t\right)=\hat{\psi}\left(\left[e_{1}, t\right]^{\mathcal{V}}\right)-e_{1} \cdot \hat{\psi}(t)+t \cdot \hat{\psi}\left(e_{1}\right)=0 \\
& \Leftrightarrow-\left[e_{1}, \hat{\psi}(t)\right]^{\mathcal{W}}=-c\left[e_{1}, e_{0}\right]^{\mathcal{W}}=c e_{1}=0 \\
& \Leftrightarrow c=0 .
\end{aligned}
$$

Inserting $\hat{\psi}(t)=0$ into A.1 , we obtain

$$
0=\left(\delta_{1}^{\mathcal{V}} \hat{\psi}\right)\left(x_{1}, x_{2}\right)=\left(\delta_{1}^{\mathcal{W}} \psi\right)\left(x_{1}, x_{2}\right) .
$$

This means that a cocycle $\hat{\psi} \in Z^{1}(\mathcal{V}, \mathcal{W})$ corresponds to a cocycle $\psi \in Z^{1}(\mathcal{W}, \mathcal{W})$ when projected to $\mathcal{W}$. Moreover, a cocycle $\psi$ of $Z^{1}(\mathcal{W}, \mathcal{W})$ can also be lifted to a cocycle $\hat{\psi}:=\psi \circ \nu$ in $Z^{1}(\mathcal{V}, \mathcal{W})$. By definition, we thus have $\hat{\psi}(t)=0$ and the relation A.2 holds true. Hence, a cocycle $\psi \in Z^{1}(\mathcal{W}, \mathcal{W})$ yields a cocycle $\hat{\psi} \in Z^{1}(\mathcal{V}, \mathcal{W})$ and we have $Z^{1}(\mathcal{V}, \mathcal{W}) \cong Z^{1}(\mathcal{W}, \mathcal{W})$ in a canonical way.

The second step of the proof consists in comparing the coboundaries of $B^{1}(\mathcal{V}, \mathcal{W})$ and those 
of $B^{1}(\mathcal{W}, \mathcal{W})$. However, this is trivial. In fact, the coboundary condition applied on a 0 cochain $\phi \in C^{0}(\mathcal{V}, \mathcal{W})$ is the same as the one applied on a 0 -cochain $\phi \in C^{0}(\mathcal{W}, \mathcal{W})$, yielding in both cases:

$$
\left(\delta_{0} \phi\right)(x)=-x \cdot \phi \text { with } \phi \in \mathcal{W} .
$$

Since the central element of $\mathcal{V}$ acts trivially on $\mathcal{W}$, we have $B^{1}(\mathcal{V}, \mathcal{W}) \cong B^{1}(\mathcal{W}, \mathcal{W})$. All in all, we conclude $\mathrm{H}^{1}(\mathcal{V}, \mathcal{W}) \cong \mathrm{H}^{1}(\mathcal{W}, \mathcal{W})$ in a canonical way.

\section{REFERENCES}

[1] Arbarello, E., deConcini, C., Kac, V., Procesi, C., Moduli spaces of Curves and representation theory, Comm. Math. Phys. 117 (1988), 1-36. MR 946992

[2] Elie Cartan, Les groupes de transformations continus, infinis, simples, Ann. Sci. École Norm. Sup. (3) 26 (1909), 93-161. MR 1509105

[3] Claude Chevalley and Samuel Eilenberg, Cohomology theory of Lie groups and Lie algebras, Trans. Amer. Math. Soc. 63 (1948), 85-124. MR 0024908

[4] Jill Ecker, The low-dimensional algebraic cohomology of infinite-dimensional Lie algebras of Virasoro type, Ph.D. thesis, 2020, to appear.

[5] Jill Ecker and Martin Schlichenmaier, The Vanishing of the Low-Dimensional Cohomology of the Witt and the Virasoro algebra, (2017), arXiv:1707.06106.

[6] _ The Low-Dimensional Algebraic Cohomology of the Virasoro Algebra, (2018), arXiv:1805.08433.

[7] _ The low-dimensional algebraic cohomology of the Witt and the Virasoro algebra, J. Phys. Conf. Ser. 1194 (2019), no. 1, 012032.

[8] Alice Fialowski, Deformations of some infinite-dimensional Lie algebras, J. Math. Phys. 31 (1990), no. 6, 1340-1343. MR 1054321

[9] _ Formal rigidity of the Witt and Virasoro algebra, J. Math. Phys. 53 (2012), no. 7, 073501, 5, arXiv:1202.3132, DOI:10.1063/1.4731220. MR 2985241

[10] Alice Fialowski and Martin Schlichenmaier, Global deformations of the Witt algebra of Krichever-Novikov type, Commun. Contemp. Math. 5 (2003), no. 6, 921-945. MR 2030563

[11] D. B. Fuks, Cohomology of infinite-dimensional Lie algebras, Contemporary Soviet Mathematics, Consultants Bureau, New York, 1986, Translated from the Russian by A. B. Sosinskiǔ. MR 874337

[12] I. M. Gelfand and D. B. Fuks, Cohomologies of the Lie algebra of vector fields on the circle, Funkcional. Anal. i Priložen. 2 (1968), no. 4, 92-93. MR 0245035

[13] Murray Gerstenhaber, The cohomology structure of an associative ring, Ann. of Math. (2) 78 (1963), 267-288. MR 0161898

[14] _ On the deformation of rings and algebras, Ann. of Math. (2) 79 (1964), 59-103. MR 0171807

[15] _ A uniform cohomology theory for algebras, Proc. Nat. Acad. Sci. U.S.A. 51 (1964), 626-629. MR 0160807

[16] On the deformation of rings and algebras. II, Ann. of Math. 84 (1966), 1-19. MR 0207793

[17] $\quad$ On the deformation of rings and algebras. III, Ann. of Math. (2) 88 (1968), 1-34. MR 0240167 
[18] L. V. Goncharova, Cohomology of Lie algebras of formal vector fields on the line, Uspehi Mat. Nauk 27 (1972), no. 5(167), 231-232. MR 0402765

[19] G. Hochschild and J.-P. Serre, Cohomology of group extensions, Trans. Amer. Math. Soc. 74 (1953), 110-134. MR 0052438

[20] _ Cohomology of Lie algebras, Ann. of Math. (2) 57 (1953), 591-603. MR 0054581

[21] Victor G. Kac, Ashok K. Raina, and Natasha Rozhkovskaya, Bombay lectures on highest weight representations of infinite dimensional Lie algebras, second ed., Advanced Series in Mathematical Physics, vol. 29, World Scientific Publishing Co. Pte. Ltd., Hackensack, NJ, 2013. MR 3185361

[22] John McCleary, A user's guide to spectral sequences, second ed., Cambridge Studies in Advanced Mathematics, vol. 58, Cambridge University Press, Cambridge, 2001. MR 1793722

[23] Valentin Ovsienko and Claude Roger, Generalizations of Virasoro group and Virasoro algebra through extensions by modules of tensor-densities on $S^{1}$, Indag. Math. (N.S.) 9 (1998), no. 2, 277-288. MR 1691416

[24] V. N. Reshetnikov, The cohomology of two Lie algebras of vector fields on the circle, Uspehi Mat. Nauk 26 (1971), no. 1(157), 231-232. MR 0292097

[25] Martin Schlichenmaier, An elementary proof of the formal rigidity of the Witt and Virasoro algebra, Geometric methods in physics, Trends Math., Birkhäuser/Springer, Basel, 2013, pp. 143-153. MR 3363999

[26] _ An elementary proof of the vanishing of the second cohomology of the Witt and Virasoro algebra with values in the adjoint module, Forum Math. 26 (2014), no. 3, 913-929, arXiv:1111.6625, DOI:10.1515/forum-2011-0143. MR 3200354

[27] Toru Tsujishita, On the continuous cohomology of the Lie algebra of vector fields, Proc. Japan Acad. Ser. A Math. Sci. 53 (1977), no. 4, 134-138. MR 0458517

[28] N. W. van den Hijligenberg and Yu. Yu. Kotchetkov, The absolute rigidity of the NeveuSchwarz and Ramond superalgebras, J. Math. Phys. 37 (1996), no. 11, 5858-5868. MR 1417181

[29] Friedrich Wagemann, Explicit formulae for cocycles of holomorphic vector fields with values in $\lambda$ densities, J. Lie Theory 11 (2001), no. 1, 173-184. MR 1828289

[30] _ Infinite dimensional Lie algebras - cohomology and deformations, Habilitation à diriger des recherches, Université de Nantes, November 2007.

[31] Charles A. Weibel, An introduction to homological algebra, Cambridge Studies in Advanced Mathematics, vol. 38, Cambridge University Press, Cambridge, 1994. MR 1269324

[32] Lin Sheng Zhu and Dao Ji Meng, Some infinite-dimensional complete Lie algebras, Chinese Ann. Math. Ser. A 21 (2000), no. 3, 311-316. MR 1775845

University of Luxembourg, Mathematics Research Unit, Faculty of Science, Technology and Communication, Campus Belval, Maison du Nombre, 6, avenue de la Fonte, L-4364 EschSur-Alzette, LuXembourg

Email address: jill.ecker@uni.lu

University of Luxembourg, Mathematics Research Unit, Faculty of Science, Technology and Communication, Campus Belval, Maison du Nombre, 6, avenue de la Fonte, L-4364 EschSur-Alzette, Luxembourg

Email address: martin.schlichenmaier@uni.lu 